\title{
Baroclinic Instability on Hot Extrasolar Planets
}

\author{
I. Polichtchouk ${ }^{\star}$ and J. Y-K. Cho* \\ Astronomy Unit, School of Physics and Astronomy, Queen Mary University of London, London E1 4NS, UK
}

Accepted 2012 May 15. Received 2012 March 21; in original form 2011 December 31

\begin{abstract}
We investigate baroclinic instability in flow conditions relevant to hot extrasolar planets. The instability is important for transporting and mixing heat, as well as for influencing large-scale variability on the planets. Both linear normal mode analysis and non-linear initial value calculations are carried out - focusing on the freely-evolving, adiabatic situation. Using a highresolution general circulation model (GCM) which solves the traditional primitive equations, we show that large-scale jets similar to those observed in current GCM simulations of hot extrasolar giant planets are likely to be baroclinically unstable on a timescale of few to few tens of planetary rotations, generating cyclones and anticyclones that drive weather systems. The growth rate and scale of the most unstable mode obtained in the linear analysis are in qualitative, good agreement with the full non-linear calculations. In general, unstable jets evolve differently depending on their signs (eastward or westward), due to the change in sign of the jet curvature. For jets located at or near the equator, instability is strong at the flanks - but not at the core. Crucially, the instability is either poorly or not at all captured in simulations with low resolution and/or high artificial viscosity. Hence, the instability has not been observed or emphasized in past circulation studies of hot extrasolar planets.
\end{abstract}

Key words: hydrodynamics - planets and satellites: atmospheres - methods: numerical instabilities - turbulence - waves

\section{INTRODUCTION}

Baroclinic instability is a generic flow instability that occurs in rotating, stably-stratified fluids subject to a meridional (northward) temperature gradient. Examples of such a fluid are planetary atmospheres and oceans. The temperature gradient induces a vertical (altitudinal) shear in the mean flow by thermal wind balance (e.g. Pedlosky 1987); hence, baroclinic flows are those that nominally vary in the vertical direction. The instability itself is important because it gives rise to large- and meso-scale weather systems on planets. It also serves as a source of turbulence, which has been invoked as the initial condition in some simulations of extrasolar planets to generate plausible initial jet profiles (e.g. Cho et al. 2003, 2008). More importantly, the instability is a source of spatio-temporal variability which could be observed remotely.

Baroclinic instability on extrasolar planets has not been studied thus far. In this work we perform a simple linear analysis of a horizontally uniform jet in vertical shear. We also use a highlyaccurate pseudospectral general circulation model (GCM) which solves the hydrostatic primitive equations to study the non-linear evolution of a non-uniform, gradient-wind balanced jet on an extrasolar planet. The primitive equations solved govern the large-scale dynamics of planetary atmospheres (e.g. Holton 1992; see also Cho et al. 2008 for some discussion relevant to the present work). Here

\footnotetext{
^ E-mail: I.Polichtchouk@qmul.ac.uk; J.Cho@qmul.ac.uk
}

$\dagger$ Corresponding author the main focus is on close-in gaseous planets, as they remain the best studied type of extrasolar planets thus far. However, much of the findings and discussion apply to hot extrasolar planets in general - regardless of whether a solid boundary is present or the radiatively stable layer extends deeply into the planet.

For concreteness, we present calculations for a model planet with physical parameters appropriate for the extrasolar giant planet HD209458b (Table 1). We focus on the stability of broad, highspeed zonal jets - positive (eastward) at the equator and negative (westward) at high latitude - under adiabatic (i.e. heating and cooling rates balanced in the net) situation. By 'broad' we mean a width of $\sim L_{\mathrm{D}}$, where $L_{\mathrm{D}}$ is the Rossby deformation length (section 2.1), and 'high-speed' means the speed is $\sim 1000 \mathrm{~m} \mathrm{~s}^{-1}$ at the core of the jet. Such jets are commonly produced in diabatically-forced GCM simulations of close-in extrasolar giant planets (e.g. Showman et al. 2008; Rauscher \& Menou 2010; Thrastarson \& Cho 2010). A study of adiabatic behaviour is needed because it provides the necessary baseline for comparing the instability under forced conditions and because, in many circumstances, the produced jets are not maintained by the applied thermal forcing (but some flow-modified version, away from the specified radiative equilibrium).

Our basic approach in this work is to carefully study baroclinic instability in sufficient generality, without complicating the fundamental process with details which are still uncertain for extrasolar planets. The primary aim here is three-fold: 1) to ascertain the importance of baroclinic instability as a generic process operating on extrasolar planets; 2) to gain a better understanding of the outputs 


\section{I. Polichtchouk and J. Y-K. Cho}

from current extrasolar planet GCM simulations, made difficult by the complexity of solving the primitive equations accurately; and, 3 ) to explore fundamental issues in baroclinic instability that have received less emphasis in traditional geophysical fluid dynamics studies, due to the markedly different parameter regime of many extrasolar planets compared to that of the Earth.

The overall plan of the paper is as follows. Section 2 presents linear stability analysis. Linear growth rates and phase speeds are calculated for the traditional primitive equations on the ' $\beta$-plane', a differentially rotating plane tangent to the surface of the planet at a given latitude. In section 3 we present the non-linear evolution of the instability, obtained from full numerical simulations. This section also presents the description of the numerical model and setup, as well as the non-convergence of under-resolved and/or over-dissipated simulations. The foundation for baroclinic lifecycle study is also laid in this section; a detailed discussion of the phenomenon is presented elsewhere, as are of forced evolution and transient growth. Recapitulation and discussion are given in section 4.

\section{LINEAR THEORY}

\subsection{Charney-Stern-Pedlosky Criteria}

Necessary conditions for instability exist. These may be derived directly from global conservation of pseudoenergy and are given in Charney \& Stern (1962) and Pedlosky (1964). Hence, we shall not derive the conditions here but simply list them for the reader's convenience. The conditions play an important role in this work, particularly in understanding the setup of the nonlinear initial value problem (section 3$)$.

Let $q=\alpha(\boldsymbol{\omega}+2 \boldsymbol{\Omega}) \cdot \nabla^{\mathrm{L}} \varphi$ be the potential vorticity, where $\boldsymbol{\omega}$ is the relative vorticity, $\boldsymbol{\Omega}$ is the planetary vorticity, $\alpha$ is the specific volume $\left(=1 / \rho\right.$, where $\rho$ is the density) and $\nabla^{\mathrm{L}}$ is a gradient operator acting on a materially conserved field $\varphi$, which may be a function of temperature and pressure (e.g. potential temperature or entropy). Additionally, let $x, y$ and $z$ be the zonal (i), meridional $(\mathbf{j})$ and vertical $(\mathbf{k})$ directions, respectively. Given the zonal flow, $\mathbf{U}=U(y, z) \mathbf{i}$, and the basic state potential vorticity $Q(y, z)$ such that $q(x, y, z)=Q+q^{\prime}(x, y, z)$, one of the following necessary criteria must be met for the onset of instability:

(i) $\partial Q / \partial y$ and $\partial U / \partial z$ are opposite signs at the upper boundary

(ii) $\partial Q / \partial y$ and $\partial U / \partial z$ are same signs at the lower boundary

(iii) $\partial U / \partial z$ is the same sign at the upper and lower boundaries - a condition that is distinct from condition (i) or (ii), if $\partial Q / \partial y=0$

(iv) $\partial Q / \partial y$ changes sign somewhere in the interior.

Note that $Q=Q(\mathbf{U})$ and the prime denotes deviation from the basic state.

For realistic flow profiles studied in section 3, the instability criterion is normally satisfied through criterion (iv). In addition, criteria (i) and (ii) are also satisfied in most cases. These conditions are useful for assessing stability of any basic flow configuration. However, they do not provide quantitative information, such as the growth rates of unstable modes and phase speeds of waves/eddies generated by the instability.
Table 1. Numerical parameter values for HD209458b. Here $g$ is surface gravity; $R_{p}$ is equatorial radius; $\Omega$ is rotation rate; $\mathcal{R}$ is gas constant; $c_{p}$ is specific heat at constant pressure; $H$ is scale height; $p_{\mathrm{r}}$ is reference pressure; $T_{\text {eq }}$ is equilibrium temperature; $U$ is characteristic flow speed; and, $N$ is Brunt-Väisälä frequency.

\begin{tabular}{lll}
\hline \hline Parameter & Value & Units \\
\hline$g$ & 9.8 & $\mathrm{~m} \mathrm{~s}^{-2}$ \\
$R_{p}$ & $10^{8}$ & $\mathrm{~m}$ \\
$\Omega$ & $2.1 \times 10^{-5}$ & $\mathrm{~s}^{-1}$ \\
$\mathcal{R}$ & $3.5 \times 10^{3}$ & $\mathrm{~J} \mathrm{~kg}^{-1} \mathrm{~K}^{-1}$ \\
$c_{p}$ & $1.23 \times 10^{4}$ & $\mathrm{~J} \mathrm{~kg}^{-1} \mathrm{~K}^{-1}$ \\
$H$ & $5.8 \times 10^{5}$ & $\mathrm{~m}$ \\
$p_{\mathrm{r}}$ & $10^{5}$ & $\mathrm{~Pa}$ \\
$T_{\text {eq }}$ & 1500 & $\mathrm{~K}$ \\
$U$ & 1000 & $\mathrm{~m} \mathrm{~s}^{-1}$ \\
$N$ & $2 \times 10^{-3}$ & $\mathrm{~s}^{-1}$ \\
\hline \hline
\end{tabular}

For the Earth, the stability analysis is typically based on the quasi-geostrophic (QG) theory, in which small Rossby number Ro and order unity Burger number $B u$ are assumed (Charney 1947; Eady 1949; Phillips 1951). Given the characteristic flow speed $U$, Coriolis parameter $f$, horizontal length scale $L$, and the Rossby deformation length scale $L_{\mathrm{D}}, R o$ and $B u$ are defined $R o=U /(f L)$ and $B u=\left(L_{\mathrm{D}} / L\right)^{2}$, respectively. Here $L_{\mathrm{D}}=N H / f$ is the Rossby deformation length, where $N$ is the Brunt-Väisälä frequency; $H$ is the characteristic vertical scale; and, $f=2 \Omega \sin \phi$, where $\Omega=|\boldsymbol{\Omega}|$ is the planetary rotation rate and $\phi$ is the latitude. Note that both $R o$ and $B u$ vary with latitude. For example, formally, $R o \rightarrow \infty$ as $\phi \rightarrow 0$.

In QG theory, adiabatic dynamics is governed by the material advection of potential vorticity:

$$
\frac{\mathrm{D} q_{\mathrm{QG}}}{\mathrm{D} t}=0,
$$

where $\mathrm{D} / \mathrm{D} t$ is the material derivative and

$$
q_{\mathrm{QG}}=f+\nabla^{2} \psi+\alpha f_{0}^{2} \frac{\partial}{\partial z}\left(\frac{1}{\alpha N^{2}} \frac{\partial \psi}{\partial z}\right)
$$

is the QG potential vorticity in the ' $\beta$-plane approximation' (see section 2.3). Here $f=f_{0}+\beta y$, where $f_{0}=f\left(\phi_{0}\right)$ and $\beta=$ $\left.(\mathrm{d} f / \mathrm{d} y)\right|_{\phi=\phi_{0}}$ for a specific latitude $\phi_{0} ; \psi$ is the streamfunction; and, $\nabla^{2}$ is the horizontal Laplacian operator. Note that $q_{\mathrm{QG}}$ can be inverted - as with the full primitive equation $q$, under the QG balance condition - to obtain all other dynamical variables (Hoskins, McIntyre \& Robertson 1985).

The QG equations (the above advection equation for $q_{\mathrm{QG}}$ plus boundary conditions) derive from the more complete primitive equations (e.g. Pedlosky 1987). The standard QG equations are appropriate for large-scale motions on many planets, away from the low latitudes. However, they are not broadly ${ }^{1}$ appropriate for a large number of extrasolar planets, which are characterized by $R O$ of order unity (see Table 1) - even away from the equatorial region. More importantly, much dynamics of interest on extrasolar planets occur in the equatorial region (section 3.5), where the traditional QG theory does formally break down. Therefore, we perform our stability analysis using the full primitive equations.

${ }^{1}$ QG theory may still be valid locally on hot extrasolar planets. 


\subsection{Governing Equations}

In the standard pressure ( $p$ ) coordinate system (Kasahara 1974), the hydrostatic primitive equations read:

$$
\begin{aligned}
& \frac{\partial \mathbf{v}}{\partial t}+\mathbf{v} \cdot \tilde{\nabla} \mathbf{v}+\omega \frac{\partial \mathbf{v}}{\partial p}+f \mathbf{k} \times \mathbf{v}+\tilde{\nabla} \Phi=\mathcal{F}_{\mathbf{v}} \\
& \frac{\partial \theta}{\partial t}+\mathbf{v} \cdot \tilde{\nabla} \theta+\omega \frac{\partial \theta}{\partial p}=\mathcal{F}_{\theta} \\
& \tilde{\nabla} \cdot \mathbf{v}+\frac{\partial \omega}{\partial p}=0 \\
& \frac{\partial \Phi}{\partial p}+h \theta=0 .
\end{aligned}
$$

Here $\mathbf{v}(\mathbf{x}, t)=(u, v)$ is the (zonal, meridional) velocity in the frame rotating with $\Omega$, where $\mathbf{x} \in \mathbb{R}^{3} ; \omega=\tilde{\mathrm{D}} p / \tilde{\mathrm{D}} t$ is the 'vertical' velocity, where $\tilde{\mathrm{D}} / \tilde{\mathrm{D}} t=\partial / \partial t+\mathbf{v} \cdot \tilde{\nabla}+\omega \partial / \partial p$ with $\tilde{\nabla}$ operating along constant surfaces of $p$ (which in general is not materially conserved); $\theta=T\left(p_{\mathrm{r}} / p\right)^{\kappa}$ is the potential temperature, where $T$ is the temperature, $p_{\mathrm{r}}$ is the reference pressure, $\kappa=\mathcal{R} / c_{p}$ with $\mathcal{R}$ the gas constant and $c_{p}$ the specific heat at constant pressure; $\Phi=g z$ is the geopotential above the planetary radius $R_{p}$, where $g$ is the constant surface gravity; $h(p)=\mathcal{R}\left(p / p_{\mathrm{r}}\right)^{\kappa} / p$; and, $\mathcal{F}_{\mathbf{v}}$ and $\mathcal{F}_{\theta}$ are, respectively, momentum and potential temperature sources/dissipations. From here on, we exclusively work in $p$-coordinate and drop the tilde over the advective and gradient operators for notational clarity.

The above set of equations is closed with the ideal gas law, $p \alpha=\mathcal{R} T$. The equations are also supplemented with the boundary conditions,

$$
\omega=0 \quad \text { at } \quad p=0, p_{\mathrm{r}} .
$$

Hence, the domain boundaries are material surfaces and no mass flows across them.

\subsection{Two-Layer, Beta-Plane Analysis}

In this section, we linearize equations (1) on the $\beta$-plane, where $f(\phi)$ is represented by $f(y)=f_{0}+\beta y$. Here, $f_{0}$ and $\beta$ are constants, $y=R_{p}\left(\phi-\phi_{0}\right)$ and the motion is assumed to be periodic in the zonal direction with no meridional component at the latitudinal boundaries. The $\beta$-plane is a tangent plane located at $\phi_{0}$, and the setup is only formally justified for scales that are small compared to $R_{p}$. However, in practice the $\beta$-plane approximation mainly results in small distortion of planetary waves and captures the essential qualitative behavior. For the analysis in this section, we neglect source/dissipation terms in equations (1) - i.e. $\mathcal{F}_{\mathbf{v}}(\mathbf{x}, t)=\mathcal{F}_{\theta}(\mathbf{x}, t)=0$. This is because, as discussed in section 1, we are interested in the dynamics of jets that result from conditions e.g. when the net heating is not large or the effective thermal relaxation time is not small.

We perform a standard normal mode analysis of the baroclinic instability admitted by a two-layer representation of equations (1). Similar work has been carried out by Wiin-Nielsen (1963) and Fraedrich \& Frisius (2001) for the Earth. As in these studies, we simplify equation set (1) to that appropriate for a discretised model with two equally-spaced, stacked layers in the $p$-coordinate. In this model $\mathbf{v}, \theta$ and $\Phi$ are defined at odd levels and $\omega$ is defined at even levels. The structure is illustrated in Fig. 1. The equations for the

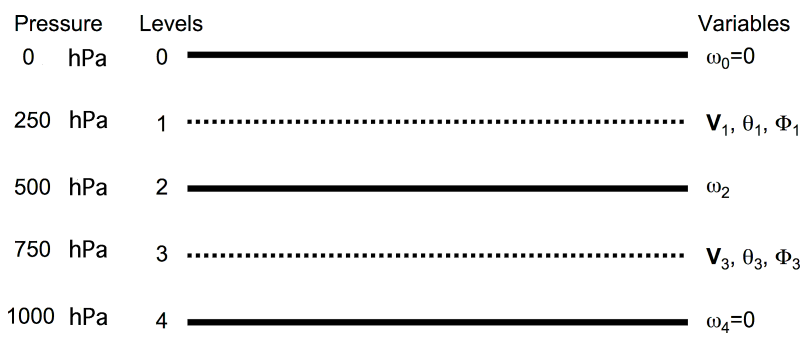

Figure 1. Vertical structure of the two-layer primitive equations model. Different field variables are defined on different levels; $1 \mathrm{hPa}=10^{2} \mathrm{~Pa}$ $=1$ mbar. Bold lines are the layer boundaries.

interior levels are:

$$
\begin{aligned}
& \frac{\partial \mathbf{v}_{1}}{\partial t}+\mathbf{v}_{1} \cdot \nabla \mathbf{v}_{1}+\omega_{2}\left(\frac{\mathbf{v}_{3}-\mathbf{v}_{1}}{2 \triangle p}\right)+f \mathbf{k} \times \mathbf{v}_{1}=-\nabla \Phi_{1} \\
& \frac{\partial \mathbf{v}_{3}}{\partial t}+\mathbf{v}_{3} \cdot \nabla \mathbf{v}_{3}+\omega_{2}\left(\frac{\mathbf{v}_{3}-\mathbf{v}_{1}}{2 \triangle p}\right)+f \mathbf{k} \times \mathbf{v}_{3}=-\nabla \Phi_{3} \\
& \frac{\partial \theta_{1}}{\partial t}+\nabla \cdot\left(\theta_{1} \mathbf{v}_{1}\right)+\frac{\omega_{2} \theta_{2}}{\triangle p}=0 \\
& \frac{\partial \theta_{3}}{\partial t}+\nabla \cdot\left(\theta_{3} \mathbf{v}_{3}\right)-\frac{\omega_{2} \theta_{2}}{\triangle p}=0 \\
& \nabla \cdot \mathbf{v}_{1}+\frac{\omega_{2}}{\triangle p}=0 \\
& \nabla \cdot \mathbf{v}_{3}-\frac{\omega_{2}}{\triangle p}=0 \\
& \Phi_{1}-\Phi_{3}=h_{2} \triangle p \theta_{2},
\end{aligned}
$$

where $\triangle p=p_{\mathrm{r}} / 2$ denotes the pressure difference between odd or even numbered levels and $\theta_{2}=\theta=\left(\theta_{1}+\theta_{3}\right) / 2$. It follows from equations (3e) and (3f) that barotropic (vertically averaged) wind is non-divergent. In the present analysis, we take the Brunt-Väisälä frequency $N$ to be uniform; GCM simulations by Thrastarson \& Cho (2010) show static stability to be fairly constant over one or two scale heights for a wide range of conditions.

Baroclinic instability in the two-level primitive equations system is obtained from perturbations of an unstable 'Eady-type' basic flow with uniform vertical shear (Eady 1949):

$$
\begin{aligned}
\bar{u}_{1} & =-\bar{u}_{3}=U_{0} \\
\bar{v}_{1} & =\bar{v}_{3}=0 \\
\bar{\omega}_{1} & =\bar{\omega}_{3}=0 \\
\bar{\theta}_{1} & =-\frac{2 f_{0}}{h_{2} \triangle p} U_{0} y+\sigma_{0} \\
\bar{\theta}_{3} & =-\frac{2 f_{0}}{h_{2} \triangle p} U_{0} y-\sigma_{0} \\
\bar{\theta}_{2} & =\bar{\theta}=-\frac{2 f_{0}}{h_{2} \triangle p} U_{0} y .
\end{aligned}
$$

Here $U_{0}(=U / 2)$ characterizes the strength of the thermal wind and its shear; and, $\sigma_{0}=\left(\theta_{1}-\theta_{3}\right) / 2$ is related to the reference static stability, $S=-\alpha \partial \ln \theta / \partial p$, through

$$
S=\frac{\sigma_{0} h_{2}}{\triangle p} .
$$

For simplicity we shall consider meridionally-independent perturbations applied to the above basic flow. We now linearize equa- 
tions (3) about this basic state and arrive at the following:

$\frac{\partial \mathbf{v}_{1}^{\prime}}{\partial t}+U_{0} \frac{\partial \mathbf{v}_{1}^{\prime}}{\partial x}-\frac{U_{0}}{\triangle p} \omega_{2}^{\prime} \mathbf{i}+f \mathbf{k} \times \mathbf{v}_{1}^{\prime}=-\frac{\partial \Phi_{1}^{\prime}}{\partial x}$

$\frac{\partial \mathbf{v}_{3}^{\prime}}{\partial t}-U_{0} \frac{\partial \mathbf{v}_{3}^{\prime}}{\partial x}-\frac{U_{0}}{\triangle p} \omega_{2}^{\prime} \mathbf{i}+f \mathbf{k} \times \mathbf{v}_{3}^{\prime}=-\frac{\partial \Phi_{3}^{\prime}}{\partial x}$

$\frac{\partial \theta^{\prime}}{\partial t}-\frac{f_{0} U_{0}}{h_{2} \triangle p}\left(v_{1}^{\prime}+v_{3}^{\prime}\right)-\sigma_{0} \frac{\omega_{2}^{\prime}}{\triangle p}=0$

$\nabla \cdot \mathbf{v}_{1}^{\prime}+\frac{\omega_{2}^{\prime}}{\triangle p}=0$

$\nabla \cdot \mathbf{v}_{3}^{\prime}-\frac{\omega_{2}^{\prime}}{\triangle p}=0$

$\Phi_{1}^{\prime}-\Phi_{3}^{\prime}=h_{2} \triangle p \theta^{\prime}$.

The temperature equation $(4 \mathrm{c})$ is obtained by summing equations (3c) and (3d) and linearising. Further, if we denote the vertical average of a variable $\xi$ by

$$
\xi_{+} \equiv \frac{1}{2}\left(\xi_{1}+\xi_{3}\right)
$$

and the half vertical difference by

$$
\xi_{-} \equiv \frac{1}{2}\left(\xi_{1}-\xi_{3}\right)
$$

summing and differencing equations (4a) and (4b) give:

$$
\begin{aligned}
& \frac{\partial \mathbf{v}_{+}^{\prime}}{\partial t}+U_{0} \frac{\partial \mathbf{v}_{-}^{\prime}}{\partial x}-\frac{U_{0}}{\triangle p} \omega_{2}^{\prime} \mathbf{i}+f \mathbf{k} \times \mathbf{v}_{+}^{\prime}=-\frac{\partial \Phi_{+}^{\prime}}{\partial x} \\
& \frac{\partial \mathbf{v}_{-}^{\prime}}{\partial t}+U_{0} \frac{\partial \mathbf{v}_{+}^{\prime}}{\partial x}+f \mathbf{k} \times \mathbf{v}_{-}^{\prime}=-\frac{\partial \Phi_{-}^{\prime}}{\partial x} .
\end{aligned}
$$

By applying curl and divergence, we obtain vorticity and divergence forms, respectively, of the above equations. The equations set is closed when potential temperature and pressure velocity are eliminated using the hydrostatic and continuity equations. We then introduce the streamfunctions, $\psi_{1}$ and $\psi_{3}$, and the velocity potentials, $\chi_{1}$ and $\chi_{3}$, for levels 1 and 3 such that

$$
\frac{\partial^{2}}{\partial x^{2}}\left(\chi_{1}+\chi_{3}\right)=0
$$

and obtain four evolution equations for the barotropic vorticity, baroclinic vorticity, baroclinic divergence, and geopotential (i.e. potential temperature):

$$
\begin{aligned}
\frac{\partial^{2} \psi_{+}^{\prime}}{\partial x^{2}} & =\frac{\partial^{2}}{\partial x^{2}}\left(\frac{\psi_{1}^{\prime}+\psi_{3}^{\prime}}{2}\right), \\
\frac{\partial^{2} \psi_{-}^{\prime}}{\partial x^{2}} & =\frac{\partial^{2}}{\partial x^{2}}\left(\frac{\psi_{1}^{\prime}-\psi_{3}^{\prime}}{2}\right), \\
\frac{\partial^{2} \chi_{-}^{\prime}}{\partial x^{2}} & =\frac{\partial^{2}}{\partial x^{2}}\left(\frac{\chi_{1}^{\prime}-\chi_{3}^{\prime}}{2}\right)=-\frac{\omega_{2}^{\prime}}{\triangle p} \\
\Phi_{-}^{\prime} & =\frac{h_{2} \triangle p \theta^{\prime}}{2}
\end{aligned}
$$

respectively. The evolution equations for these quantities are:

$$
\begin{aligned}
\frac{\partial}{\partial t}\left(\frac{\partial^{2} \psi_{+}^{\prime}}{\partial x^{2}}\right) & =-U_{0} \frac{\partial}{\partial x}\left(\frac{\partial^{2} \psi_{-}^{\prime}}{\partial x^{2}}\right)-\beta \frac{\partial \psi_{+}^{\prime}}{\partial x} \\
\frac{\partial}{\partial t}\left(\frac{\partial^{2} \psi_{-}^{\prime}}{\partial x^{2}}\right) & =-U_{0} \frac{\partial}{\partial x}\left(\frac{\partial^{2} \psi_{+}^{\prime}}{\partial x^{2}}\right)-f_{0} \frac{\partial^{2} \chi_{-}^{\prime}}{\partial x^{2}}-\beta \frac{\partial \psi_{-}^{\prime}}{\partial x} \\
\frac{\partial}{\partial t}\left(\frac{\partial^{2} \chi_{-}^{\prime}}{\partial x^{2}}\right) & =-\frac{\partial^{2} \Phi_{-}^{\prime}}{\partial x^{2}}+f_{0} \frac{\partial^{2} \psi_{-}^{\prime}}{\partial x^{2}}-\beta \frac{\partial \chi_{-}^{\prime}}{\partial x} \\
\frac{\partial \Phi_{-}^{\prime}}{\partial t} & =U_{0} f_{0} \frac{\partial \psi_{+}^{\prime}}{\partial x}-\frac{\mathcal{R} \sigma_{0}}{2^{\kappa+1}} \frac{\partial^{2} \chi_{-}^{\prime}}{\partial x^{2}} .
\end{aligned}
$$

At this point, the foregoing system of equations can be made non-dimensional for a more 'generalized' treatment, as is typical in instability studies. However, we shall describe our analysis of the equations presented in the dimensional form. We feel this facilitates a more lucid interpretation of the results in some ways. For the interested reader, we have included the non-dimensional account in Appendix A and refer the reader to that section, especially for the dependence of the results on non-dimensional parameters.

Denoting disturbances by

$$
\boldsymbol{\Psi}=\hat{\boldsymbol{\Psi}} \exp \{i k(x-c t)\}
$$

where $\boldsymbol{\Psi}=\left(\psi_{+}^{\prime}, \psi_{-}^{\prime}, \chi_{-}^{\prime}, \Phi_{-}^{\prime}\right)^{\mathrm{T}}, \hat{\boldsymbol{\Psi}}=\left(\hat{\Psi}_{+}, \hat{\Psi}_{-}, \hat{\chi}_{-}, \hat{\Phi}_{-}\right)^{\mathrm{T}}$ and $c \in \mathbb{C}$, equations (6) reduce to

$$
\mathbf{M} \hat{\boldsymbol{\Psi}}=0
$$

with

$$
\mathbf{M}=\left[\begin{array}{cccc}
-c-\beta / k^{2} & U_{0} & 0 & 0 \\
U_{0} & -c-\beta / k^{2} & -i f_{0} / k & 0 \\
0 & i f_{0} / k & -c-\beta / k^{2} & -i / k \\
f_{0} U_{0} & 0 & -i k \mathcal{R} \sigma_{0} / 2^{\kappa+1} & c
\end{array}\right]
$$

For a non-trivial solution, $\operatorname{det}(\mathbf{M})=0$. This leads to a fourth-order characteristic equation for $c$ :

$$
\begin{aligned}
& c^{4}+c^{3}\left(\frac{3 \beta}{k^{2}}\right)+c^{2}\left(\frac{3 \beta^{2}}{k^{4}}-\frac{f_{0}^{2}}{k^{2}}-\frac{\mathcal{R} \sigma_{0}}{2^{\kappa+1}}-U_{0}^{2}\right)+ \\
& c\left(\frac{\beta^{3}}{k^{6}}-\frac{\beta f_{0}^{2}}{k^{4}}-\frac{\beta \mathcal{R} \sigma_{0}}{2^{\kappa} k^{2}}-\frac{\beta U_{0}^{2}}{k^{2}}\right)+ \\
& \left(\frac{\mathcal{R} \sigma_{0} U_{0}^{2}}{2^{\kappa+1}}-\frac{f_{0}^{2} U_{0}^{2}}{k^{2}}-\frac{\beta^{2} \mathcal{R} \sigma_{0}}{2^{\kappa+1} k^{4}}\right)=0 .
\end{aligned}
$$

Equation (7) is solved numerically for $c$ as a function of $k$, while keeping the values of $f_{0}, \beta, U_{0}, \mathcal{R}, \sigma_{0}$ and $\kappa$ constant. If $\Im \mathfrak{m}\{c\} \neq 0$, the disturbances grow or decay exponentially since they are proportional to $\exp \{-i k c t\}$. Two of the roots of equation (7) are stable eastward- and westward-traveling inertia-gravity waves. The other two roots are baroclinic waves. These waves propagate neutrally (i.e. without growing or decaying) eastward and westward, if $\Im \mathfrak{m}\{c\}=0$ (provided $\Re \mathfrak{e}\{c\} \neq 0$ ).

The baroclinic wave solutions to equation (7) are presented in Fig. 2 for a planet with HD209458b parameters given in Table 1 . The left panel shows the growth rate, $k \cdot \Im \mathfrak{m}\{c\}$, as a function of wavelength $2 \pi k^{-1}$ at several different latitudes: $\phi=$ $\left(60^{\circ}, 45^{\circ}, 35^{\circ}, 25^{\circ}\right)$. The growth rates are labelled 'HD60' (red), 'HD45' (green), 'HD35' (yellow) and 'HD25' (blue), respectively. The right panel shows the corresponding phase wave speeds $\Re \mathfrak{e}\{c\}$. Note here that unstable baroclinic waves travel westward relative to the mean flow $(\Re \mathfrak{e}\{c\}<0)$.

From Fig. 2 it can be seen that the wavelength of the most unstable mode at $\phi=60^{\circ}$ is $1.7 \times 10^{8} \mathrm{~m}$, corresponding to 1.8 undulations (i.e. $\sim 2$ crests and troughs each) at this latitude. The growth rate of the instability is $3.1 \tau^{-1}$, where $\tau=2 \pi \Omega^{-1}$ is the planetary rotation time. At $\phi=45^{\circ}$ and $\phi=35^{\circ}$, the most unstable modes correspond to 2.2 and 2.3 undulations around their respective latitude circles and with growth rates $2.3 \tau^{-1}$ and $1.5 \tau^{-1}$, respectively. Hence, jets centred at lower latitudes have increased growth times with modestly increased wavelengths of the most unstable mode. Significantly, linear analysis predicts stability for jets 
Growth Rate $\times 10^{-6} \mathrm{~s}^{-1}$

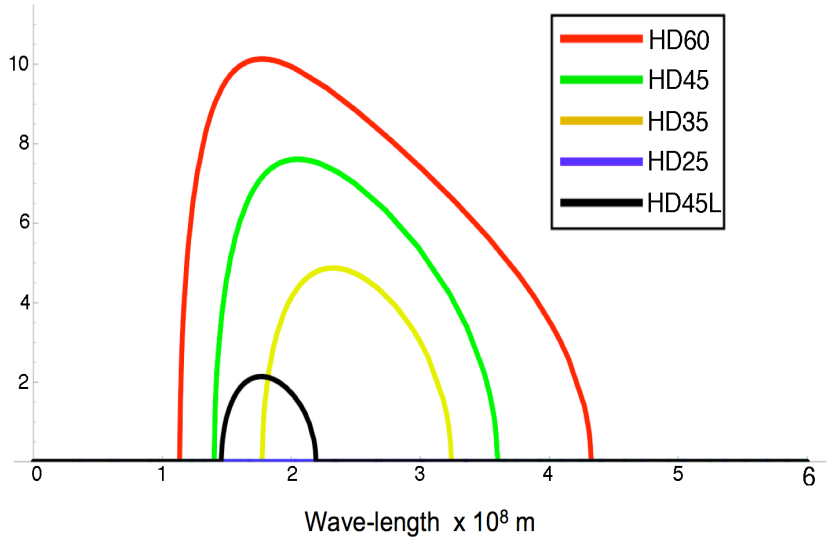

Phase Speed $\mathrm{m} \mathrm{s}^{-1}$

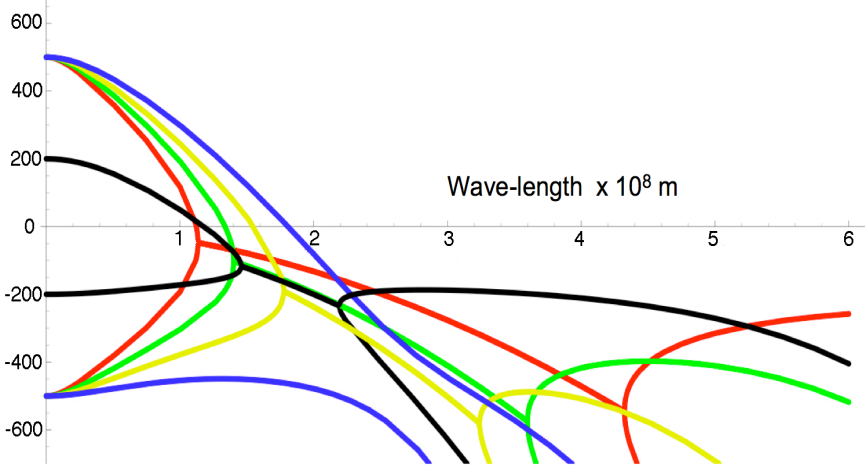

Figure 2. Growth rate $[k \cdot \Im \mathfrak{m}\{c\}]$ (left) and phase speed [Re $\{c\}]$ (right) for HD209458b, as a function of wavelength $2 \pi k^{-1}$. Curves 'HD60', 'HD45', 'HD35' and 'HD25' represent growth rates and phase speeds at $\phi=\left(60^{\circ}, 45^{\circ}, 35^{\circ}, 25^{\circ}\right) ; f_{0}=4.2 \times 10^{-5} \sin \phi \mathrm{s}^{-1}, \beta=4.2 \times 10^{-13} \cos \phi \mathrm{m}^{-1} \mathrm{~s}^{-1}$, $U_{0}=500 \mathrm{~m} \mathrm{~s}^{-1}, \mathcal{R}=3500 \mathrm{~J} \mathrm{~kg}^{-1} \mathrm{~K}^{-1}, \sigma_{0}=300 \mathrm{~K}$ and $\kappa=0.286$. Curve 'HD45L' has been computed for HD209458b parameters at $\phi=45^{\circ}$, but with $U_{0}=200 \mathrm{~m} \mathrm{~s}^{-1}$.

Growth Rate $x 10^{-6} \mathrm{~s}^{-1}$

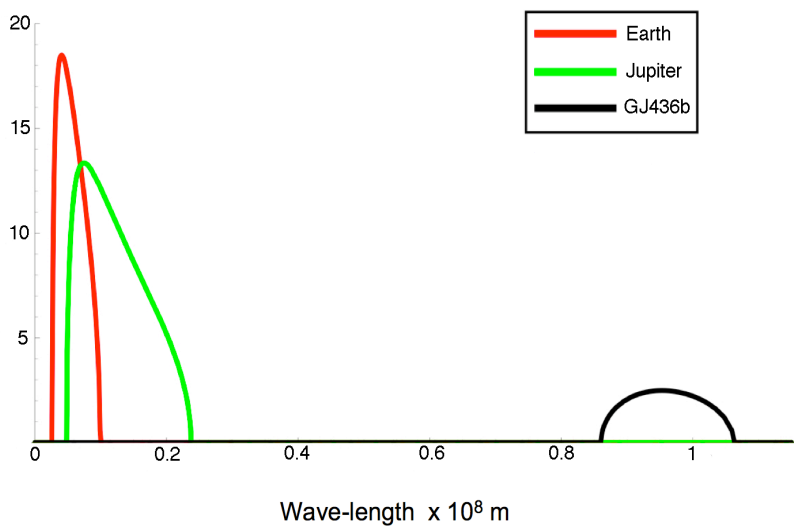

Phase Speed $\mathrm{m} \mathrm{s}^{-1}$

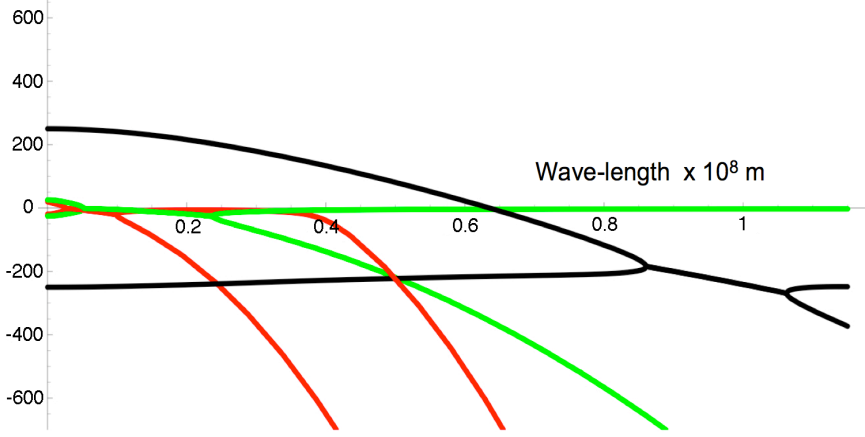

Figure 3. Growth rate $[k \cdot \Im \mathfrak{m}\{c\}]$ (left) and phase speed $[\Re \mathfrak{e}\{c\}]$ (right) at $\phi=45^{\circ}$ for Earth, Jupiter and GJ436b as a function of wavelength $2 \pi k^{-1}$. For the Earth, $f_{0}=10^{-4} \mathrm{~s}^{-1}, \beta=1.6 \times 10^{-11} \mathrm{~m}^{-1} \mathrm{~s}^{-1}, U_{0}=20 \mathrm{~m} \mathrm{~s}^{-1}, \mathcal{R}=287 \mathrm{~J} \mathrm{~kg}^{-1} \mathrm{~K}^{-1}, \sigma_{0}=15 \mathrm{~K}, \kappa=0.286$. For Jupiter, $f_{0}=2.5 \times 10^{-4} \mathrm{~s}^{-1}, \beta=3.5 \times 10^{-12} \mathrm{~m}^{-1} \mathrm{~s}^{-1}, U_{0}=25 \mathrm{~m} \mathrm{~s}^{-1}, \mathcal{R}=3779 \mathrm{~J} \mathrm{~kg}^{-1} \mathrm{~K}^{-1}, \sigma_{0}=24 \mathrm{~K}, \kappa=0.286$. For GJ436b, $f_{0}=3.9 \times 10^{-5} \mathrm{~s}^{-1}$, $\beta=1.4 \times 10^{-12} \mathrm{~m}^{-1} \mathrm{~s}^{-1}, U_{0}=250 \mathrm{~m} \mathrm{~s}^{-1}, \mathcal{R}=3500 \mathrm{~J} \mathrm{~kg}^{-1} \mathrm{~K}^{-1}, \sigma_{0}=150 \mathrm{~K}, \kappa=0.286$. Note, $\sigma_{0}$ values for Jupiter and GJ436b have been computed assuming constant temperatures of $120 \mathrm{~K}$ and $750 \mathrm{~K}$, respectively. Note the change in scales, compared with Fig. 2.

located at or equator-ward of $\phi=28^{\circ}$ (see e.g. the flat, blue curve labelled 'HD25').

To illustrate the dependence of the growth rate and phase speed on the characteristic flow speed (or, equivalently, shear strength), we also present in Fig. 2 result obtained for the case with $U_{0}=200 \mathrm{~m} \mathrm{~s}^{-1}$ at $\phi=45^{\circ}$ (black curve labelled 'HD45L'). Comparing the 'HD45' (green) and 'HD45L' (black) curves, we see immediately that the growth rate of the most unstable mode decreases significantly for the smaller $U_{0}$ case. The instability takes $\sim 4$ times longer to develop in the weaker speed/shear case. We also note that the wavelength of the most unstable mode decreases slightly.
Hence, as $U_{0}$ decreases, the number of undulations increases for a jet located at a given latitude.

The qualitative behaviour described above is not restricted to HD209458b. It applies to any planet that has a meridional temperature gradient. To illustrate the general applicability of our results, we present in Fig. 3 the growth rates and phase speeds at $\phi=45^{\circ}$ for several planets: Earth, Jupiter and GJ436b (red, green and black curves, respectively). For the Earth, the wavelength of the most unstable mode is $4100 \mathrm{~km}$, corresponding to $\sim 7$ undulations at midlatitude, with growth rate of $1.6 \tau^{-1}$ (i.e. growth time of 15 hours). This is consistent with many studies of baro- 
clinic instability on the Earth (e.g. Thorncroft et al. 1993; Polvani et al. 2004). The corresponding values of undulations for Jupiter and GJ436b are $\sim 43$ and $\sim 1$ with growth rates $0.48 \tau^{-1}$ and $0.56 \tau^{-1}$, respectively. Accordingly, if baroclinic instability occurs on these planets, it appears Jupiter simulations must be of very high resolution to capture the instability. On the other hand, the instability at the midlatitude of GJ436b would clearly be of planetary scale and thus may lead to a possible observable variability signal for this planet on a timescale of $\sim 1.8$ planetary rotations. Note that the phase speeds of the unstable baroclinic waves on Earth and Jupiter are very small (close to zero) compared to those on the extrasolar planets, HD209458b and GJ436b.

We have also carried out linear growth rate analysis with the two-layer QG model for HD209458b and have compared the results with those from the primitive equations model, presented above. In the two models, the growth rates at high latitudes and midlatitudes are equivalent to within 5 per cent. However, at low latitudes, the QG model overestimates the growth rates by approximately 25 per cent. Moreover, the QG model predicts instability down to $\phi=23^{\circ}$, whereas the primitive equations model predicts instability only down to $\phi=28^{\circ}$. Below these latitudes, both models predict stability. Thus, ageostrophy appears to provide a stabilizing factor in this case. Given that inertia-gravity waves are not filtered in the primitive equations model (as they are in the QG model), the enhanced stability may be due to the gravity waves 'leaking away' some of the energy that drive the instability.

\subsection{Limitations}

The preceding analysis is highly idealised. Therefore, it has limitations. For example, in general, planetary jets possess a threedimensional structure - with concurrent vertical and meridional shears, as well as zonal asymmetry. Also, the atmosphere is continuously stratified. One effect of a two-layer discretisation with uniform zonal flow in each layer is the inability to capture the symmetry breaking between eastward and westward jets. These limitations are discussed more in detail below.

In flows with both vertical and horizontal shears, the growth of unstable baroclinic waves may be suppressed by the 'barotropic governor' effect (e.g. James 1987; Nakamura 1993a; Pedlosky 1964). The effect is not fundamentally related to the sign of the jet, but a key ingredient is a counter-gradient eddy momentum flux $\overline{u^{\prime} v^{\prime}}$ generated under a horizontally sheared flow; here the overbar indicates a zonal average. The shear and the momentum flux reinforce each other to distort the meridional structure of the wave, suppressing the growth rate and shortening the wavelength of the most unstable mode. Thus, the full non-linear evolution of the instability exhibits lower growth rates and shorter wavelengths, compared with those indicated by the linear analysis presented in this section (see section 3).

The atmosphere is also continuously stratified. A representation more realistic than a two-layer model changes the instability properties described in this section. The main change is that the short-wave and long-wave cut-offs in the two-layer representation (see Figs. 2 and 3) no longer exist in the continuum of unstable modes (e.g. Charney 1947; Green 1960; Kuo 1979). The retained modes (Charney and Green modes, discussed below) are not expected to change qualitatively the asymptotic behaviour of the instability. However, they do provide additional modes for wavewave interaction during the non-linear growth phase - hence, affect the details of the evolution; this may be significant for finite-time variability.
Another limitation of the linear model presented is that it does not distinguish between the signs of the jet (or shear). This is because symmetry is preserved under the interchange of the shear sign, given the laterally uniform flow; hence, distinction between the two signs is not expected. This is in contrast to the flow used in the non-linear calculation (section 3), in which the growth rate for an unstable westward (negative shear) jet is smaller than that for the unstable eastward (positive shear) jet at the same latitude. The two signed flows behave differently in this case because of the change in the sign of the jet curvature. Furthermore, a westward jet has only one unstable mode (Charney mode) as opposed to an eastward jet, which has an infinite number of unstable modes (Green modes).

A similar observation has been made by Wang (1989), who observed a difference between eastward- and westward-sheared baroclinic flows in the Charney model (continuously stratified QG model on the $\beta$-plane). He has pointed out that the maximum growth rate for the absolute value of non-dimensional shear is substantially smaller for a flow with westward shear than a flow with eastward shear. Moreover, while the eastward jet is baroclinically unstable for any value of vertical shear $\Lambda$, the westward jet is unstable only if

$$
\Lambda<-\frac{\beta N^{2} H}{f_{0}^{2}} .
$$

Note that, at $\phi=45^{\circ}$, the critical shear for HD209458b parameters used in this work is: $\Lambda_{c}=-7.9 \times 10^{-4} \mathrm{~s}^{-1}$. The shear of the unstable westward jet described in section 3.4 is $\Lambda=-1.7 \times 10^{-3} \mathrm{~s}^{-1}$, consistent with (8).

\section{NON-LINEAR EVOLUTION}

\subsection{Numerical Model}

To study the full non-linear evolution, we use a well-tested parallel pseudospectral model, $\mathrm{BOB}^{2}$ (Scott et al. 2003). This model solves equations (1) in spherical geometry, subject to the boundary conditions (2). As in many models, the equations in the vorticitydivergence form are solved, where (relative) vorticity is $\zeta(\mathbf{x}, t)=$ $\mathbf{k} \cdot \nabla \times \mathbf{v}$ and divergence is $\delta(\mathbf{x}, t)=\nabla \cdot \mathbf{v}$. The equations in this form are more amenable for the spectral transform method (Orszag 1970; Eliasen et al. 1970; Canuto et al. 1988). Domain decomposed spectral transform algorithm is used in the horizontal direction and standard second order finite difference scheme is used in the vertical direction. The latter direction is in pressure coordinates in the numerical model.

To follow the evolution over long duration, explicit dissipation is applied so that artificial accumulation of energy at small scales is prevented (see e.g. Cho \& Polvani 1996). The dissipation is in the form of a linear superviscosity operator, $-\nu \nabla^{4}(\cdot)$, applied to the prognostic variables, $\{\zeta, \delta, \theta\}$; here $\nu$ is constant. A small RobertAsselin time filter $\epsilon$ (Robert 1966; Asselin 1972) is applied, at every time step and in each layer, to filter the computational mode arising from using a second-order time-marching scheme (see e.g. Thrastarson \& Cho 2011). No other numerical dissipators, fixers, stabilisers or filters are used in performing the simulations.

2 'Built On Beowolf' 


\subsection{Model Setup}

To study the non-linear evolution of baroclinic instability, we initialize our model with an idealized jet that satisfies the necessary condition for baroclinic instability, the Charney-Stern-Pedlosky condition described in section 2 . The jet is initially set to be either eastward or westward, and centred at a latitude between $0^{\circ}$ to $60^{\circ} \mathrm{N}$. A large number of simulations have been performed for this study, carefully varying each parameter (jet location, strength, shear, profile, direction as well as domain size, etc.) in an independent series of simulations. A very small subset of these runs, which we use for discussions in sections 3.2 to 3.5 , is given in Table 2 . The set illustrates the basic points we wish to make.

All the jets are initially non-linearly balanced so that a selfconsistent background temperature structure is generated (Fig. 4). The jets are then bumped at the beginning of the simulation by an infinitesimal temperature disturbance which is independent of altitude, a barotropic 'heat bump', and allowed to evolve freely thereafter. The setup is chosen to be similar to that in Polvani et al. (2004) for validation and comparison purposes. For example, following that work, the initial zonal flow $u_{0}$ in our runs is, in general,

$$
u_{0}(\phi, p)=\left\{\begin{array}{lc}
U \sin ^{m}\left[\pi \sin ^{2}\left(\phi-\phi_{0}\right)\right] F\left(z^{*}\right), & \phi_{0}<\phi<\phi_{\mathrm{T}} \\
0, & \text { otherwise }
\end{array}\right.
$$

Here

$$
F\left(z^{*}\right)=\frac{1}{2}\left[1-\tanh ^{3}\left(\frac{z^{*}-z_{0}}{\Delta z_{0}}\right)\right] \sin \left(\frac{\pi z^{*}}{z_{1}}\right)
$$

with $z^{*}=-H \log \left(p / p_{\mathrm{r}}\right)$, and $\phi_{0}$ and $\phi_{\mathrm{T}}$ are taken to be the following: $\phi_{0}=0$ and $\phi_{\mathrm{T}}=\pi / 2$ for jets centred at midlatitude (E45N and E45N2b), $\phi_{0}=\pi / 12$ and $\phi_{\mathrm{T}}=\pi / 2$ for jets centred at $60^{\circ} \mathrm{N}(\mathrm{W} 60 \mathrm{~N})$ and $\phi_{0}=-\pi / 4$ and $\phi_{\mathrm{T}}=\pi / 4$ for jets centred on the equator (EEQ). The typical values of the parameters are: $U= \pm 1000 \mathrm{~m} \mathrm{~s}^{-1}, z_{0}=1823 \mathrm{~km}, z_{1}=2486 \mathrm{~km}$, $\Delta z_{0}=414 \mathrm{~km}, H=580 \mathrm{~km}$, and $p_{\mathrm{r}}=10^{5} \mathrm{~Pa}(=1 \mathrm{bar})$. The latitudinal width of the jet is determined by $m$ in (9), where $m=3$ corresponds to a jet width of $\sim 40^{\circ}$ (Fig. 4a and Fig. 4b) and $m=1 / 2$ to a width of $\sim 85^{\circ}$ (Fig. 4 c). To discuss jets that closely match those produced in current GCM simulations of extrasolar giant planets, we present runs which are initialized with wider $(m=1 / 2)$ jets in the equatorial region and narrower jets $(m=3)$ poleward of $45^{\circ} \mathrm{N}$.

The basic state temperature, $T_{0}=T_{0}(\phi, p)$, is obtained by combining meridional momentum and hydrostatic balance equations:

$$
\frac{\partial T_{0}}{\partial \phi}=-\frac{H}{\mathcal{R}}\left(R_{p} f+2 u_{0} \tan \phi\right) \frac{\partial u_{0}}{\partial z^{*}} .
$$

Integrating (11) results in a temperature distribution that is in nonlinear, gradient wind balance with the specified jet. Here we have used a reference temperature of $1500 \mathrm{~K}$ as the constant of integration. The value is consistent with initial conditions and results of many GCM calculations. The basic state flow $u_{0}(\phi, p)$ and potential temperature $\theta_{0}(\phi, p)$ for runs $\mathrm{E} 45 \mathrm{~N}$ (eastward midlatitude jet), W60N (westward high latitude jet) and EEQ (wide eastward equatorial jet) are shown in Fig. 4. Recall that $\theta_{0}$ is related to $T_{0}$ by $\theta_{0}=T_{0}\left(p_{\mathrm{r}} / p\right)^{\kappa}$. To catalyse the instability, $T_{0}$ is given a small perturbation $T^{\prime}$ in the form of a localized bump at all pressure levels such that

$$
T^{\prime}(\lambda, \phi)=\mathcal{A} \operatorname{sech}^{2}\left[3\left(\lambda-\lambda_{0}\right)\right] \operatorname{sech}^{2}\left[6\left(\phi-\phi_{0}\right)\right],
$$

Table 2. Summary of jet configurations discussed: $m$ is a parameter that controls the jet width [see equation (9)]. Note, in run E45N2b the bottom boundary is set at $p=2$ bar and the vertical structure function $F\left(z^{*}\right)$ in equation (9) is specified as $F\left(z^{* *}\right)=\left\{1-\tanh ^{8}\left[\left(z^{* *}-\right.\right.\right.$ $\left.\left.\left.z_{2 \mathrm{~b}}\right) / \Delta z_{0}\right]\right\} \sin ^{4}\left(\pi z^{* *} / z_{1}\right)$, where $z^{* *}=-H \log \left[\left(p+p_{0}\right) / p_{\mathrm{r}}\right]$, with $p_{0}=60 \mathrm{hPa}$ and $z_{2 \mathrm{~b}}=900 \mathrm{~km}$.

\begin{tabular}{lccl}
\hline \hline Run & Width $(m)$ & Latitude & Direction \\
& & & \\
\hline & & & \\
E45N & 3.0 & $45^{\circ} \mathrm{N}$ & East \\
E45N2b & 3.0 & $45^{\circ} \mathrm{N}$ & East \\
W60N & 3.0 & $60^{\circ} \mathrm{N}$ & West \\
EEQ & 0.5 & $0^{\circ}$ & East \\
& & & \\
\hline \hline
\end{tabular}

for $-\pi<\lambda<\pi$. Here $\mathcal{A}=1 \mathrm{~K}$ and $\left(\phi_{0}, \lambda_{0}\right)$ represents the jet centre (latitude, longitude).

Our vertical domain, which typically extends from 1 to $10^{-3} \mathrm{bar}$, is resolved by 20 equally spaced pressure levels. The horizontal resolution of results presented in section 3.3 to section 3.5 is T170, or 170 sectoral modes and 170 total modes in the spectral expansion (see e.g. Thrastarson \& Cho 2011). The resolution is designated 'T170L20'. The inverse transformation is performed on to a $512 \times 256$ Gaussian grid covering the entire globe. The grid size is chosen for de-aliasing (Canuto et al. 1988, and references therein). Equations (1) are integrated for up to $60 \tau$ (i.e. 60 planetary rotations) with $\nu=6 \times 10^{19} \mathrm{~m}^{4} \mathrm{~s}^{-1}$. A timestep size, $\Delta t=30 \mathrm{~s}$, and a Robert-Asselin coefficient, $\epsilon=0.01$, are used for the time integration.

As already mentioned, the choice of our initial conditions is partly motivated by current GCM results of hot extrasolar giant planet atmospheres. These studies suggest typical flow speeds of $O\left(100-3000 \mathrm{~m} \mathrm{~s}^{-1}\right)$ and zonal flow consisting of up to $\sim 3$ jets often a broad equatorial eastward jet and a smaller amplitude narrower westward jet at a higher latitude on both northern and southern hemispheres (e.g. Showman et al. 2008; Rauscher \& Menou 2010; Thrastarson \& Cho 2010; Heng, Menou \& Phillips 2011). The altitudinal and latitudinal profiles used here roughly mimic those presented in fig. 9 of Showman et al. (2008) and fig. 3 of Rauscher \& Menou (2010).

In what follows, we first describe the evolution of the midlatitude eastward jet (run E45N). Although such a jet is not commonly observed in current simulations of hot extrasolar giant planets, reviewing this case is useful because it allows the present work to be compared with analogous studies - and observations - of the Earth and because it allows a baseline to be constructed for other initial conditions presented here, namely the high-latitude westward and equatorial eastward jets that match more closely with aforementioned extrasolar planet simulations.

\subsection{Paradigm Case}

Run E45N is the 'paradigm case'. It illustrates a typical non-linear evolution of a perturbed, marginally stable ${ }^{3}$ baroclinic jet on a

3 While the jets satisfy the (necessary) condition for instability, they require an initial perturbation to evolve: they are perfectly stable without the perturbation. 
a)

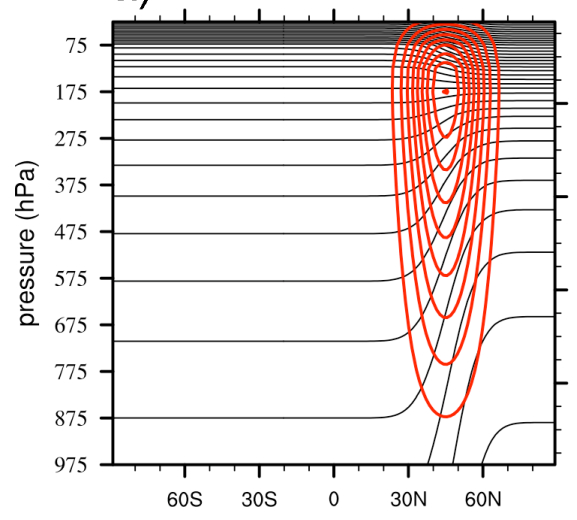

b)

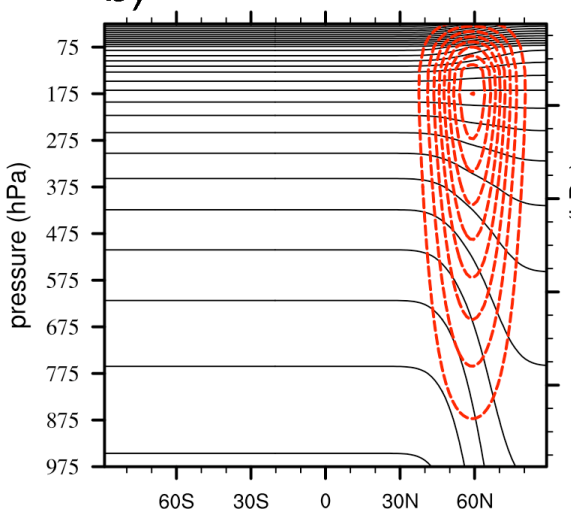

c)

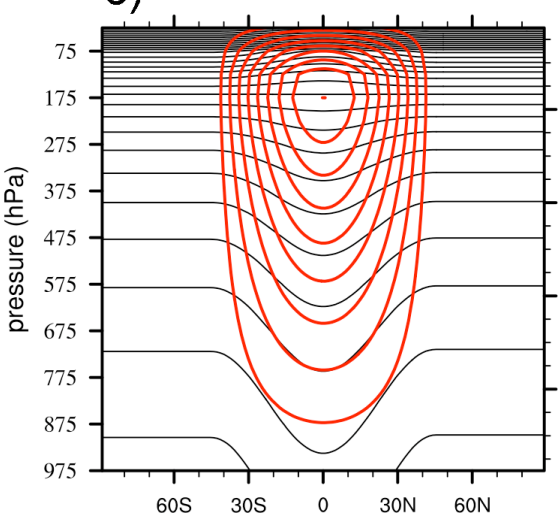

Figure 4. The basic state zonal wind $u_{0}\left[\mathrm{~m} \mathrm{~s}^{-1}\right]$ (red) and potential temperature $\theta_{0}[\mathrm{~K}]$ (black) as a function of latitude and pressure for runs (see Table 2): a) $\mathrm{E} 45 \mathrm{~N}, \mathrm{~b}) \mathrm{W} 60 \mathrm{~N}$, and c) EEQ. Contour interval for the zonal wind is $100 \mathrm{~m} \mathrm{~s}^{-1}$ and for the potential temperature $100 \mathrm{~K}$. Negative contours are dashed.

hot extrasolar giant planet in numerical simulations with high resolution. The jet is zonally symmetric and eastward with speed $1000 \mathrm{~m} \mathrm{~s}^{-1}$ at the jet core and decaying to zero at the periphery (see Fig. 4a). It meets the necessary conditions (i), (ii) and (iv) for baroclinic instability, defined in section 2, with (i) and (ii) only weakly satisfied. This can be seen from Fig. 5, which shows $\left(\partial q_{0} / \partial y\right)_{\theta}$ evaluated on an isentrope as a function of $\phi$ and $p$. Note, here $q_{0}$ is the potential vorticity defined on isobars,

$$
q_{0}(\phi, p)=-g\left(f \mathbf{k}+\nabla \times \mathbf{v}_{0}\right) \cdot \nabla \theta_{0},
$$

where $\nabla$ is the three-dimensional gradient operator in $(\lambda, \phi, p)$ space; and, $\left(\partial q_{0} / \partial y\right)_{\theta}$ is a derivative taken along an isentrope such that

$$
\left(\frac{\partial q_{0}}{\partial y}\right)_{\theta}=\left(\frac{\partial q_{0}}{\partial y}\right)_{p}-\left(\frac{\partial \theta_{0}}{\partial y}\right)_{p}\left(\frac{\partial \theta_{0}}{\partial p}\right)^{-1} \frac{\partial q_{0}}{\partial p},
$$

where $y=R_{p} \phi$ and $[\partial(\cdot) / \partial y]_{p}$ is the derivative taken on an isobar. Other cases, with jets of different sign or location, are to be compared with this one. Fig. 6 presents the evolution of $T$ (left column) and $\zeta$ (right column) fields at the $p=975 \mathrm{hPa}$ surface from run $\mathrm{E} 45 \mathrm{~N}$, for $\tau=0$ to $\tau=8$. The fields near the reference pressure level are shown since the kinetic energy is the maximum at the lower boundary for jet profiles shown in Fig. 4, similar to Gall (1976) and Simons (1972). Note that for these jets $T \approx \theta$ at this pressure level.

In Fig. 6, the perturbed jet undergoes initially a period of linear growth $(\tau \ll 4)$, when the most unstable mode emerges. At this early stage, the $T$ field shows a small-amplitude perturbation from zonal symmetry. The $\zeta$ field, on the other hand, is much more dynamic. At $\tau=4$, finite-amplitude wave breaking in the $\zeta$ field is already clearly evident, and the perturbation in this field is characterized by a distinct northwest-southeast tilt on the poleward side of the jet and southwest-northeast tilt on the equatorward side of the jet. The enhancement of the tilt proceeds concomitantly with the barotropic component of the flow, which generates negative meridional flux of the eddy zonal momentum (i.e. $\overline{u^{\prime} v^{\prime}}<0$ ) on the poleward flank of the jet and positive meridional flux of the eddy zonal momentum (i.e. $\overline{u^{\prime} v^{\prime}}>0$ ) on the equatorward flank of the jet (see e.g. Nakamura 1993b).

During this early stage of the evolution, conversion of available potential energy $(A P E)$ into eddy kinetic energy $(E K E)$ slowly

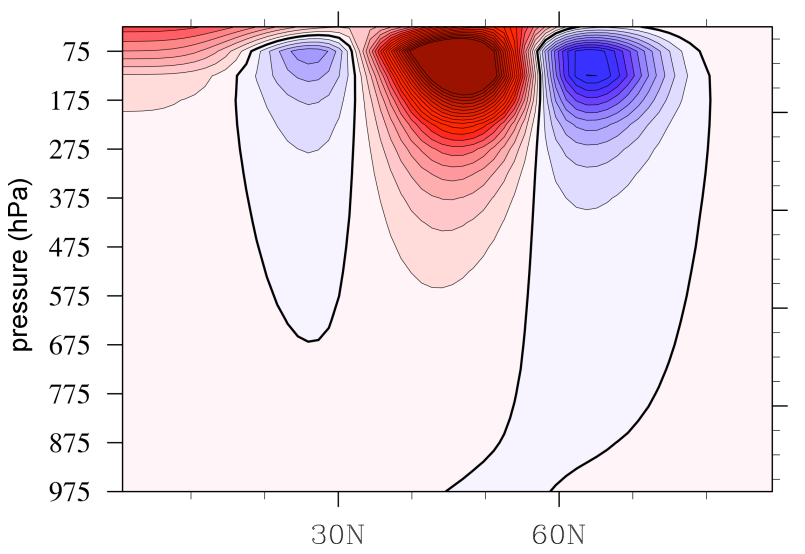

Figure 5. Meridional cross-section of the meridional potential vorticity gradient $\left(\partial q_{0} / \partial y\right)_{\theta}$ for run E45N (northern hemisphere). Maximum and minimum values are $\pm 3 \times 10^{-12} \mathrm{~K} \mathrm{~m} \mathrm{~kg}^{-1} \mathrm{~s}^{-1}$ with contour interval $2 \times 10^{-13} \mathrm{~K} \mathrm{~m} \mathrm{~kg}^{-1} \mathrm{~s}^{-1}$. Negative values are in blue and positive are in red. The zero contour is drawn with double thickness.

begins, as can be seen from Fig. 7. These quantities are defined:

$$
\begin{aligned}
& A P E=-\int_{S} \int_{0}^{p_{r}} \frac{p^{\kappa-1} \mathcal{R}}{2 g p_{r}{ }^{\kappa}}\left(\theta^{\prime \prime}\right)^{2}\left(\frac{\partial \hat{\theta}}{\partial p}\right)^{-1} d p d A \\
& E K E=\int_{S} \int_{0}^{p_{r}} \frac{1}{2 g}\left[\left(u^{\prime}\right)^{2}+\left(v^{\prime}\right)^{2}\right] d p d A,
\end{aligned}
$$

where $\theta^{\prime \prime}$ is the deviation of $\theta$ from its isobaric average $\hat{\theta}$, and the integrations are over the surface area $A$ and pressure $p$. The baroclinic instability taps the $A P E$ to drive the eddy motions.

At $\tau \approx 5$ a rapid non-linear development ensues in both fields. Note, for example, the scale change in the $\zeta$ field at $\tau=6$. A large amplitude wave can now also be clearly seen in the $T$ field. In both fields, sharp frontal features form. These dynamically-significant sharp features require very high resolution to capture faithfully. This will be discussed more in detail in section 3.6. By $\tau=8$ sharp temperature gradients trail out around the anticyclonic region (large 'clover-leaf' shaped area of negative vorticity, shaded 
0

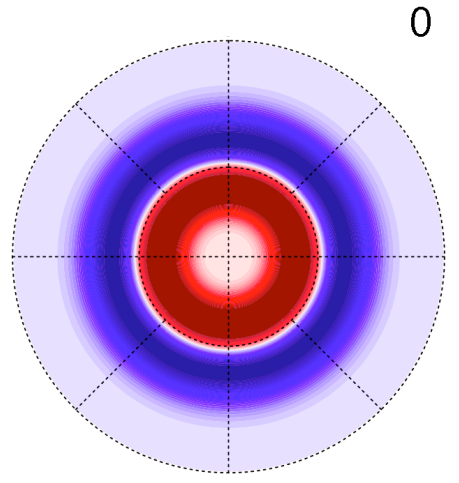

4

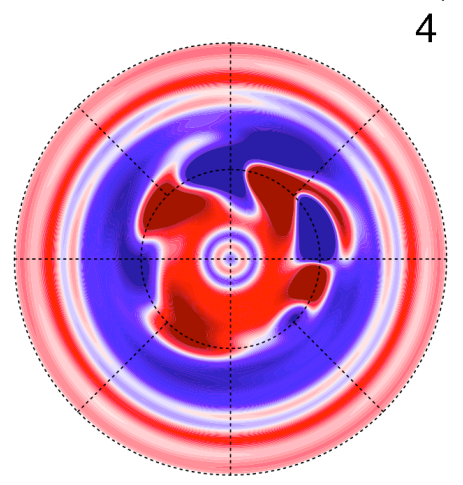

6

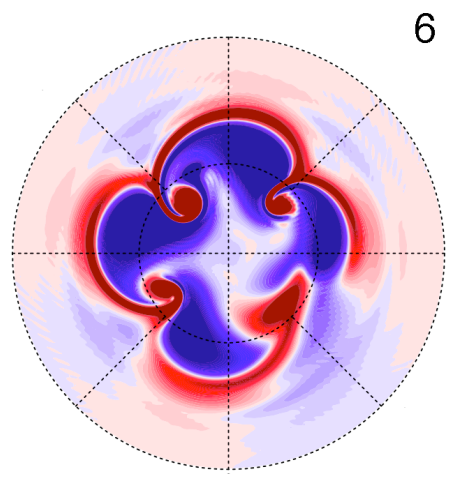

8

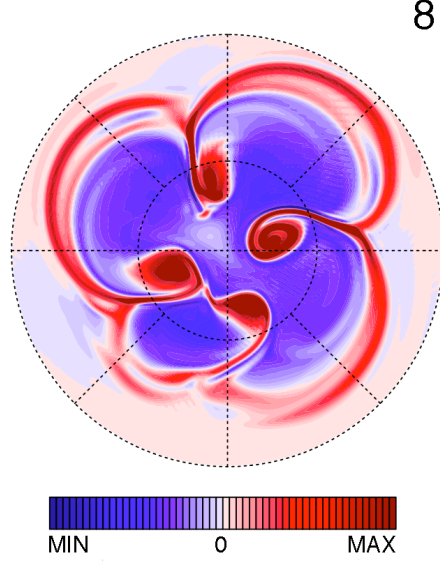

Figure 6. Temperature $T$ (left) and relative vorticity $\zeta$ (right) from run $\mathrm{E} 45 \mathrm{~N}$ in polar stereographic view, centred on the north pole. The fields are shown at the $975 \mathrm{hPa}$ pressure level for $\tau=0$ to $\tau=8$. Maximum and minimum values for $T$ are $1280 \mathrm{~K}$ and $1520 \mathrm{~K}$, respectively, with contour interval $6 \mathrm{~K}$. For $\zeta$, the maximum and minimum values are $\pm 5 \times 10^{-7} \mathrm{~s}^{-1}(\tau=0), \pm 1 \times 10^{-6} \mathrm{~s}^{-1}(\tau=4), \pm 1 \times 10^{-5} \mathrm{~s}^{-1}(\tau=6)$ and $\pm 4 \times 10^{-5} \mathrm{~s}^{-1}(\tau=8)$; the contour intervals are, respectively, $2 \times 10^{-8} \mathrm{~s}^{-1}, 4 \times 10^{-8} \mathrm{~s}^{-1}, 4 \times 10^{-7} \mathrm{~s}^{-1}$ and $1.6 \times 10^{-6} \mathrm{~s}^{-1}$. The spectral resolution of this simulation is T170L20 (see text). Note the large, nearly two orders of magnitude, change in the magnitude of $\zeta$ during the evolution - as well as the formation of sharp fronts and coherent vortices, particularly at $\tau=6$ and $\tau=8$. 


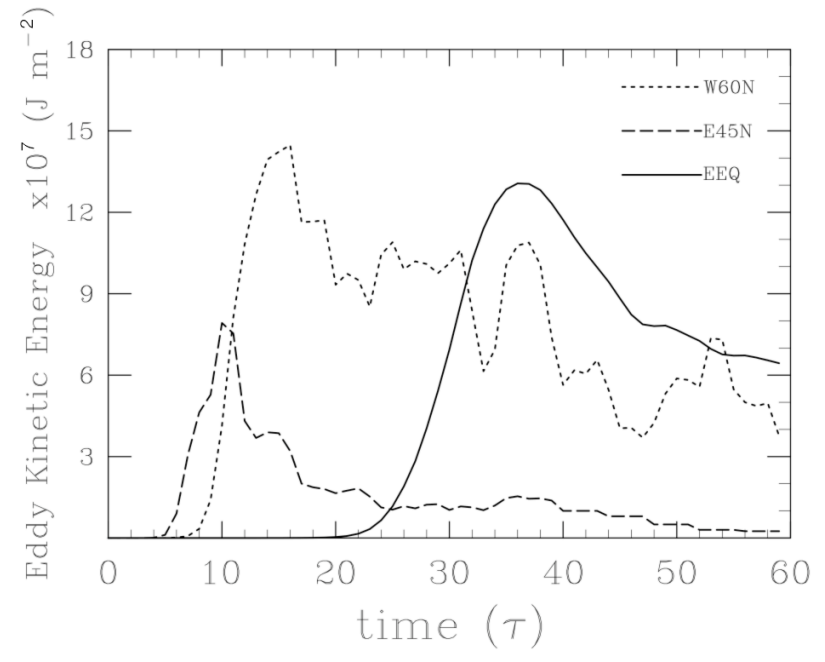

Figure 7. Evolution of globally-averaged eddy kinetic energy (per area) $\left[\mathrm{J} \mathrm{m}^{-2}\right]$ for runs E45N (dashed line), W60N (dotted line) and EEQ (solid line). The eddy kinetic energy for EEQ has been multiplied by a factor of 50.

in blue), forming curved baroclinic fronts. At this time, the $E K E$ is well into its non-linear growth stage. Note the pools of warm air that have been pinched off (cyclonic vortices embedded in the anticyclonic region), intruding into the high latitudes. Simultaneously, broad regions of cool air spread into the tropical region from higher latitude (i.e. negative heat transport). Thus, the original equatorpole temperature gradient is significantly reduced by the instability.

The poleward heat transport can be checked against linear theory for eddy transport (see e.g. Holton 1992; Vallis 2006) by examining the zonal cross-sections of streamfunction, meridional velocity and temperature perturbations: $\psi^{\prime}, v^{\prime}$ and $T^{\prime}$, respectively. The cross-sections at midlatitude are shown in Fig. 8. As can be seen, $\psi^{\prime}$ and $v^{\prime}$ tilt westward with height and $T^{\prime}$ tilts eastward with height, demonstrating that heat transport is taking place. Note that, in the case of baroclinically unstable westward jet at the same latitude, the directions of the tilts are reversed. This is because gradient wind balance produces a temperature distribution that is warmer at the poles than at the equator, as can be seen in Fig. 4b. This results in an equatorward transport of heat by the eddies (section 3.4).

The long-time evolution of the run presented in Fig. 6 is illustrated in Fig. 9 ( $\tau=10$ and $\tau=40)$. By $\tau \approx 40$ the $T$ and $\zeta$ fields have organised into essentially zonal structures and eddy activity has mostly ceased. The cyclones that have emerged from the baroclinic wave breaking, strongly interact $(\tau=10)$ and ultimately merge into an unsteady cyclonic polar vortex $(\tau=40)$. A similar 'end-state', resulting from vortex mergers, has been observed in HD209458b simulations of Cho et al. (2003).

The long-range interaction of the like-signed vortices on hot extrasolar planets is more pronounced than on the Earth (and other cool, rapidly-rotating planets). This can be explained by the much larger Rossby deformation length scale, $L_{\mathrm{D}} / R_{p}=\mathcal{O}(1)$, on the hot extrasolar giant planet. Larger $L_{\mathrm{D}}$ means more robust mergers and a more dynamic final vortex (Cho et al. 2003, 2008; Cho \& Polvani 1996). Scott (2011) has recently quantified this behaviour: merger and poleward migration of cyclones ensues if the potential vorticity anomaly $q^{\prime}$ associated with a vortex exceeds the magnitude of the planetary vorticity $2 \Omega$ by $\sim 12$ per cent. In our case,
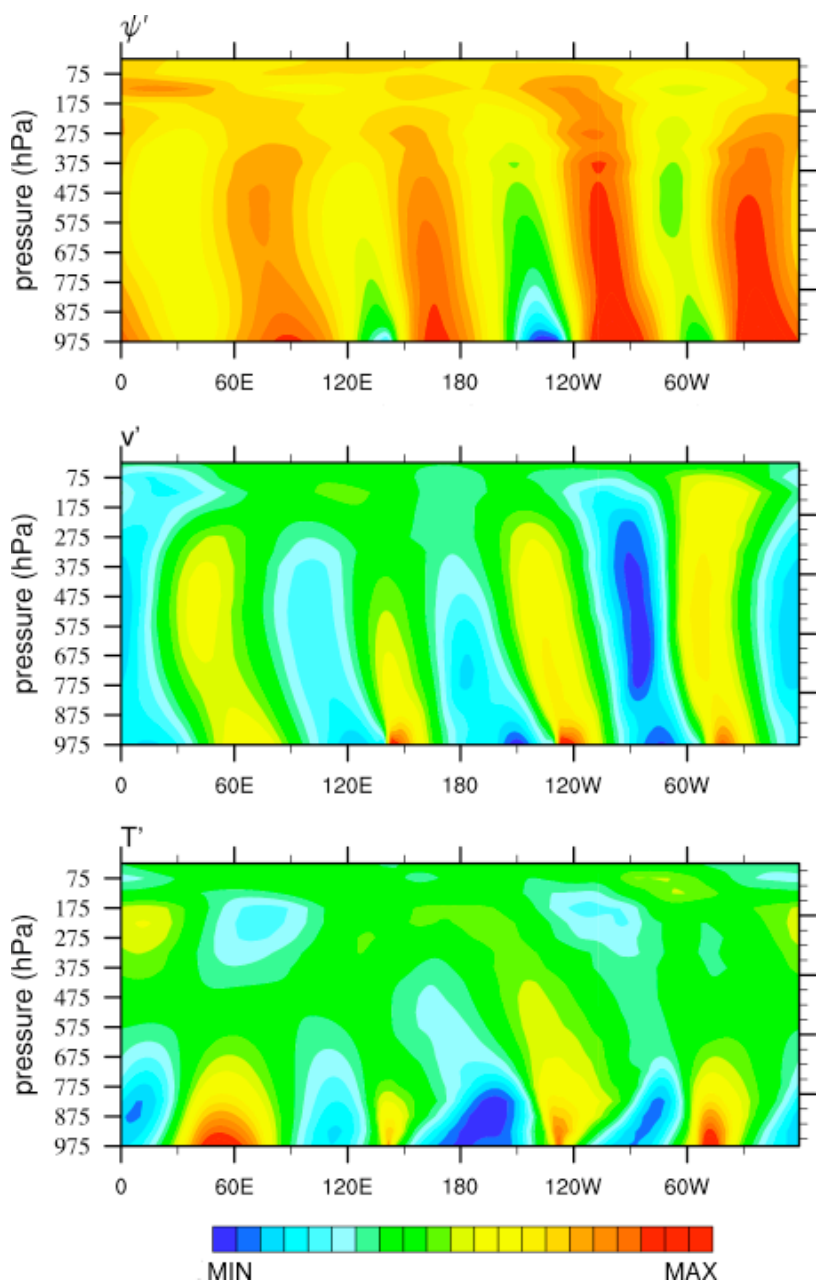

Figure 8. Perturbation streamfunction $\psi^{\prime}$ (top), perturbation meridional velocity $v^{\prime}$ (middle) and perturbation temperature $T^{\prime}$ (bottom) at midlatitude as a function of pressure and longitude at $\tau=6$ for run E45N. Contour intervals are: $-24 \times 10^{8} \mathrm{~m}^{2} \mathrm{~s}^{-1}$ to $11 \times 10^{8} \mathrm{~m}^{2} \mathrm{~s}^{-1}$ in steps of $10^{8} \mathrm{~m}^{2} \mathrm{~s}^{-1}$, $-180 \mathrm{~m} \mathrm{~s}^{-1}$ to $320 \mathrm{~m} \mathrm{~s}^{-1}$ in steps of $20 \mathrm{~m} \mathrm{~s}^{-1}$ and $-75 \mathrm{~K}$ to $125 \mathrm{~K}$ in steps of $5 \mathrm{~K}$, respectively. Note, $\psi^{\prime}$ and $v^{\prime}$ tilt westward with height and $T^{\prime}$ tilts eastward with height, signifying meridional transport of heat and reduction of equator-pole temperature gradient.

we find $\left(q^{\prime} / 2 \Omega\right) \geqslant 1.19$ - i.e. anomaly excess of 19 per cent - by $\tau=6$, consistent with Scott's finding.

The temporal evolution of the global average EKE (the dashed line for run E45N and solid line for run EEQ in Fig. 7) is typically described as a 'baroclinic growth - barotropic decay' cycle (e.g. Simmons \& Hoskins 1979; Thorncroft et al. 1993). During the cycle, conversion of $A P E$ to $E K E$ is impeded by a positive feedback between the horizontal shear in the flow and the eddy momentum flux. At $\tau \geqslant 10$, the disturbances in run $\mathrm{E} 45 \mathrm{~N}$ are sheared out and $E K E$ is lost to the mean flow through the Reynolds stresses more than it is gained through baroclinic conversion. The feedback is the main component in the previously mentioned nonlinear 'barotropic governor effect', affected by the horizontal shear in the jet, spherical geometry and ageostrophy (see e.g. Nakamura 1993b).

The zonal mean zonal wind $\bar{u}$ and zonal mean zonal potential 

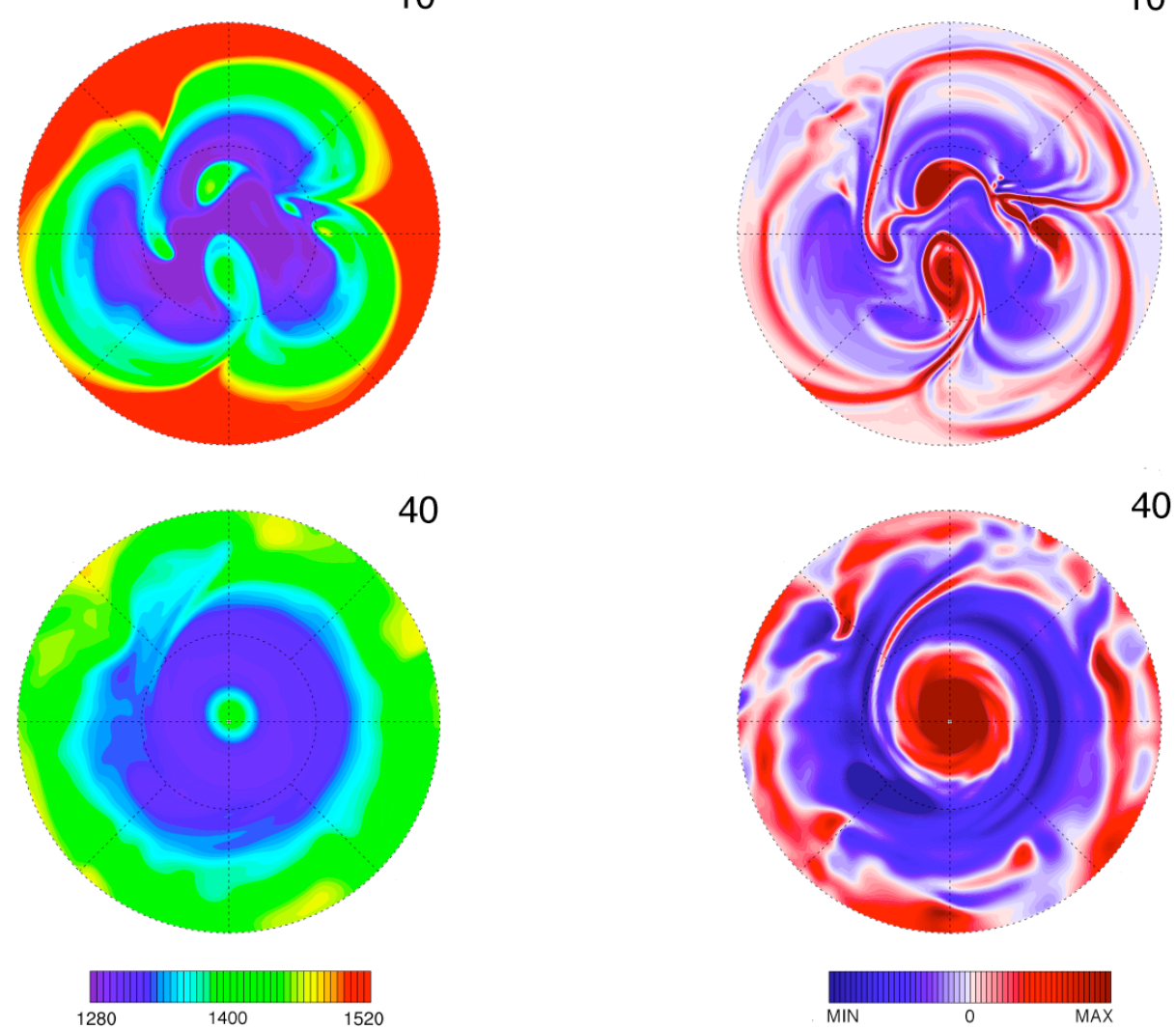

Figure 9. Same as Fig. 6 but for $\tau=10$ and $\tau=40$. Contour interval for temperature is $6 \mathrm{~K}$. The maximum and minimum contours for relative vorticity are $\pm 4 \times 10^{-5} \mathrm{~s}^{-1}$ at $\tau=10$ and $\pm 10^{-5} \mathrm{~s}^{-1}$ at $\tau=40$. The respective contour intervals are $1.6 \times 10^{-6} \mathrm{~s}^{-1}$ and $4 \times 10^{-7} \mathrm{~s}^{-1}$.

a)

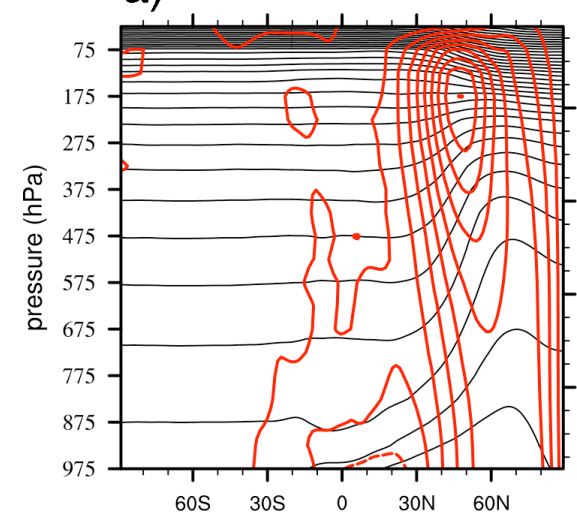

b)

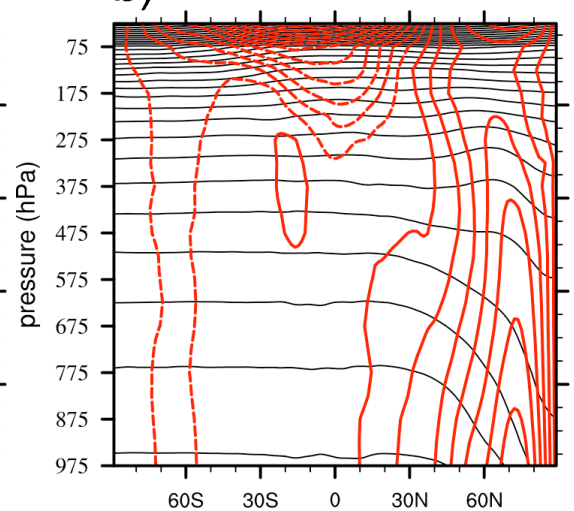

c)

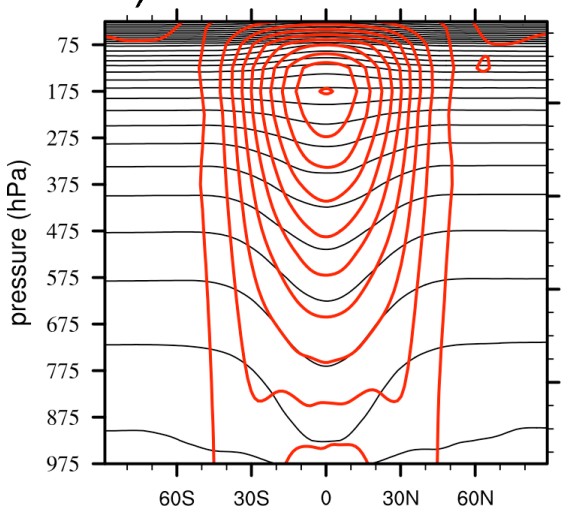

Figure 10. Zonal mean zonal wind $\bar{u}$ (red) and potential temperature $\bar{\theta}$ (black) contours for runs a) E45N at $\tau=30, \mathrm{~b}$ ) W60N at $\tau=60$ and c) EEQ at $\tau=60$. Wind contour interval for runs E45N and EEQ is $100 \mathrm{~m} \mathrm{~s}^{-1}$ and for run W60N is $50 \mathrm{~m} \mathrm{~s}^{-1}$. Temperature contour interval is $100 \mathrm{~K}$. Negative (westward) wind contours are dashed. 
temperature $\bar{\theta}$ at the end of the life-cycle is presented in Fig. 10 (see panel $a$ on the left for run E45N). The jet itself has becomes broader and more barotropic - much like the 'LC1 life cycle' reported in Thorncroft et al. (1993). Furthermore, the meridional entropy gradient $\mathrm{d} \bar{\theta} / \mathrm{d} \phi$ is significantly reduced compared to the initial state, particularly at the lower levels in the domain (cf. Fig. 4a). Much of the $A P E$ is taken up by the kinetic energy of the zonal mean flow and the flow is accelerated there. To quantify the accelerations, consider the transformed Eulerian-mean zonal momentum equation (e.g. Andrews \& McIntyre 1978):

$$
\begin{aligned}
\frac{\partial \bar{u}}{\partial t}=-\left[\frac{1}{R_{p} \cos \phi} \frac{\partial}{\partial \phi}(\bar{u} \cos \phi)\right. & -f] \bar{v}^{*}-\frac{\partial \bar{u}}{\partial p} \bar{\omega}^{*} \\
& +\frac{1}{R_{p} \cos \phi} \nabla \cdot \mathbf{F} .
\end{aligned}
$$

Here $\bar{v}^{*}$ and $\bar{\omega}^{*}$ represent the 'residual' mean meridional circulation,

$$
\begin{aligned}
\bar{v}^{*} & \equiv \bar{v}-\frac{\partial}{\partial p}\left(\frac{\overline{v^{\prime} \theta^{\prime}}}{\partial \bar{\theta} / \partial p}\right) \\
\bar{\omega}^{*} & \equiv \bar{\omega}+\frac{1}{R_{p} \cos \phi} \frac{\partial}{\partial \phi}\left(\frac{\overline{v^{\prime} \theta^{\prime}}}{\partial \bar{\theta} / \partial p} \cos \phi\right),
\end{aligned}
$$

and $\mathbf{F}=\left(F_{\phi}, F_{p}\right)$ is the Eliassen-Palm (E-P) flux vector with

$$
\begin{aligned}
& F_{\phi}=R_{p} \cos \phi\left[-\overline{u^{\prime} v^{\prime}}+\left(\frac{\overline{v^{\prime} \theta^{\prime}}}{\overline{\partial \theta / \partial p}}\right)\left(\overline{\frac{\partial \bar{u}}{\partial p}}\right)\right] \\
& F_{p}=R_{p} \cos \phi\left[(\zeta+f)\left(\frac{\overline{v^{\prime} \theta^{\prime}}}{\overline{\partial \theta / \partial p}}\right)-\overline{u^{\prime} \omega^{\prime}}\right] .
\end{aligned}
$$

The influence of eddies on the mean flow is measured by the E-P fluxes: a convergent flux $(\nabla \cdot \mathbf{F}<0)$ corresponds to the deceleration of the eastward flow and a divergent flux $(\nabla \cdot \mathbf{F}>0)$ corresponds to acceleration. Fig. 11 depicts vertically and temporally averaged E-P flux divergence for $\mathrm{E} 45 \mathrm{~N}$ (dashed line) over the life-cycle. The E-P fluxes are divergent in the net on the poleward flank of the jet, where the flow is accelerated, and (more strongly) convergent in the net on the equatorial flank, where overall the flow speed is reduced from the initial value (cf. Fig. 10a ).

Finally, we note that the most unstable mode for this calculation is $\sim 4$ (see Fig. 6). As discussed earlier, the linear theory of section 2.3 underestimates this number to $\sim 2$. However, the full numerical simulation shows that the simple linear theory is successful, at least qualitatively, in capturing the behavior of the instability in the following sense: the most unstable mode and the growth time for the baroclinic wave amplitude for HD209458b are smaller than the corresponding quantities for the Earth (cf., for example, Polvani et al. 2004).

\subsubsection{Lower Boundary}

As is well-known, boundary conditions are crucial in solving differential equations. Differences in the conditions, even in relatively simple physical situations, can alter the admitted solutions. For example, new or modified modes can be introduced or existing modes can be filtered by employing rigid boundary condition (i.e. $w=0$ ). The lower boundary of the simulations discussed in this paper is rigid and located at 1 bar for the most part. In this case, the vertical wind shear in the basic flow used is small, but non-zero, at the bottom boundary and baroclinically unstable modes can arise due to the presence of the boundary - via condition (ii) of the

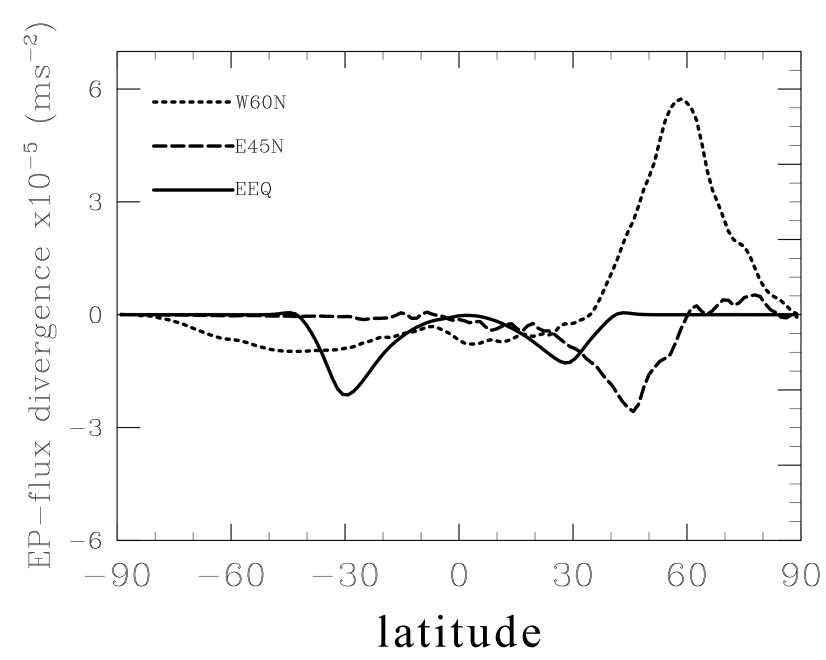

Figure 11. Vertically and temporally averaged divergence of Eliassen-Palm (E-P) flux $\left[\mathrm{m} \mathrm{s}^{-2}\right]$ for runs E45N (dashed line), W60N (dotted line) and EEQ (solid line) during the life-cycle in each run. The EEQ curve has been multiplied by a factor of 100 .

Charney-Stern-Pedlosky criteria. However, the stably-stratified radiative zone in hot extrasolar planet atmospheres may extend down to perhaps as deep as 1000 bars (Guillot \& Showman 2002). Hence, the effects of lowering the bottom boundary and preventing flow shear there require careful consideration.

Fig. 12 presents a run $(\mathrm{E} 45 \mathrm{~N} 2 \mathrm{~b})$ that is very similar to the 'paradigm case', but with the lower boundary of the calculation extended down to 2 bars. The jet is confined to pressures above the 1 bar level. In doing so we remove the influence of the lower boundary far enough away from the jet while still retaining an adequate vertical resolution. In the figure, $u_{0}$ and $\theta_{0}$ are shown in the left panel. Note, the jet profile shown in the figure has a different vertical structure than the 'paradigm case' jet. This is because balancing a jet with vertical structure given by equation (9) to the isothermal reference state produces a convectively unstable region in the computational domain, causing the vertical coordinate to lose single-valuedness and the run to immediately crash. The shown profile does not suffer from this. Significantly, the Charney-SternPedlosky criteria (iv) and (i) remain satisfied for this profile. It is crucial to understand here that, once these criteria are met, it does not matter whether the lower boundary is located at 10 or 1000 bars for the instability to occur.

The $\zeta$ field at $\tau=10$ is shown in the right panel of Fig. 12 . Although the evolution is now slightly altered from the 'paradigm case' (i.e. mode-3 is dominant, rather than mode-4), the jet is still unstable, as expected. We have verified that the evolution in run E45N2b is indeed a result of baroclinic instability: the perturbation fields tilt in the appropriate directions with height, as seen in Fig. 8 for run E45N. The instability is, however, weaker and evolves differently than when there is an initial vertical wind shear and meridional entropy gradient at the lower boundary. We have performed a simulation with the initial flow profile used in run E45N2b but with bottom raised to $1 \mathrm{bar}$, in which the shear and gradient is nonzero at the bottom. The peak global eddy kinetic energy in this run is $\sim 40$ per cent greater and vorticity perturbations are up to six times stronger than in run E45N2b. Nevertheless, the point is, the instability is present regardless of vertical flow shear at the bottom boundary. 

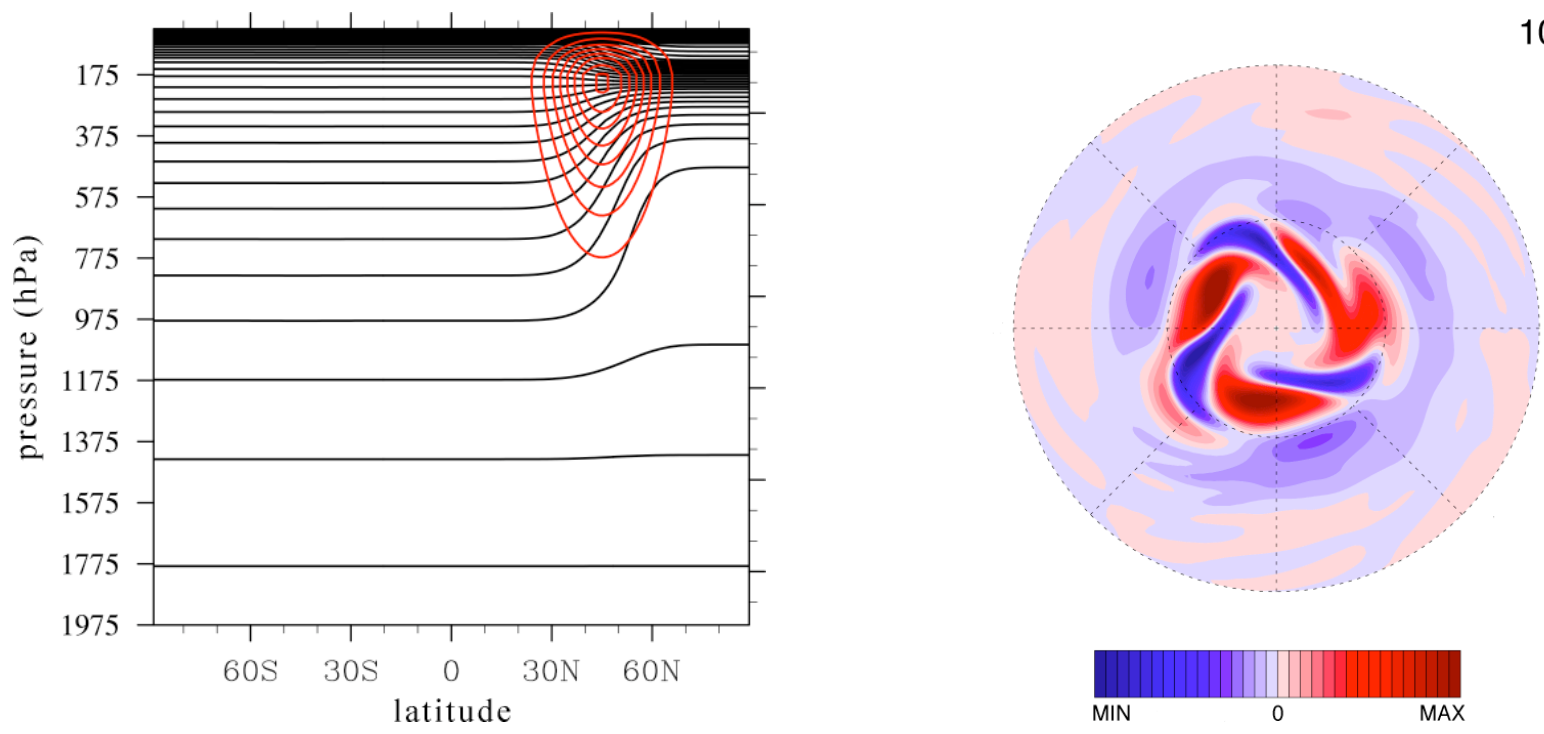

Figure 12. Left: Basic state zonal wind $u_{0}$ (red) and potential temperature $\theta_{0}$ (black) for run E45N2b with the lower boundary extended to 2 bars. Contour interval for the zonal wind is $100 \mathrm{~m} \mathrm{~s}^{-1}$ and for the potential temperature $100 \mathrm{~K}$. Right: Relative vorticity $\zeta$ from run E45N2b at $\tau=10$. The field is plotted at $975 \mathrm{hPa}$ pressure level. Maximum and minimum values are $\pm 6 \times 10^{-6} \mathrm{~s}^{-1}$, with contour intervals of $4 \times 10^{-7} \mathrm{~s}^{-1}$.

\subsection{High Latitude Westward Jet}

Having presented the evolution of the 'paradigm case', we now present the case of baroclinically unstable high-speed westward jet at high latitude (run W60N). The speed at the core of the jet is $1000 \mathrm{~m} \mathrm{~s}^{-1}$. Such jets have been observed in recent GCM simulations (e.g. Showman et al. 2008; Menou \& Rauscher 2009; Thrastarson \& Cho 2010; Heng, Menou \& Phillips 2011). In these simulations, the high latitude jets tend to be more narrow and shallow than the equatorial jets. Equatorial jets will be discussed in section 3.5. The $T$ field from run $\mathrm{W} 60 \mathrm{~N}$ at $\tau=9(975 \mathrm{hPa}$ pressure level) is shown in the left column of Fig. 13. Polar stereographic view, centred on the north pole (top frame), and cylindricalequidistant view, centred on the equator (bottom frame), are shown for latitudes poleward of $20^{\circ} \mathrm{N}$. For comparison, the right column shows the corresponding projections of the $T$ field from the E45N run at $\tau=7$, roughly at a similar stage of the evolution in run W60N.

In run W60N, baroclinic wave develops a significant northwest-southeast tilt. This is consistent with the predominantly negative momentum fluxes on the poleward side of the jet during the linear stage of the evolution. Again, the flow is characterized by sharp cyclonic fronts, this time with the most unstable mode having 3 undulations at $\phi=60^{\circ}$. The reduction in the number of undulations is also consistent with linear theory developed in section 2.3. However, contrary to the predictions from the linear analysis, the growth rate of the instability is lower than for the E45N run (cf. the onset of growth between dashed and dotted lines in Fig. 7). As already discussed, this agrees with the analysis of the Charney model by Wang (1989) and extends that result to the more general, global primitive equations model.

Note that wave-breaking in the westward jet case occurs in the opposite direction to that in the 'paradigm case' (see bottom row of Fig. 13). The waves in run W60N (left) breaks eastward, whereas in run E45N (right) the waves break westward. 'Blobs' of higher temperature fluid penetrate into the lower temperature re- gion and cooler fluid subsides into the warm region. The situation is analogous to Rayleigh-Taylor or convective instability (e.g. Sharp 1984), where a decrease of potential energy results under the interchange of two blobs at different heights. In baroclinic instability, this can occur despite the stable density stratification because the density surfaces are sloping more steeply than the line joining the two blobs. Indeed, for this reason baroclinic instability is sometimes refer to as 'sloping convection' (e.g. Vallis 2006).

Note that the EKE for the westward jet case does not follow a simple 'baroclinic growth - barotropic decay' cycle, as seen in the 'paradigm case' (Fig. 7). Instead, after the initial decay stage at $\tau \approx 15$, the $E K E$ for run $\mathrm{W} 60 \mathrm{~N}$ shows large vacillations, corresponding to a sequence of baroclinic-barotropic life cycles. Similar behavior of energetics has been observed by Feldstein (1991) for the Earth case. In a two-layer QG $\beta$-plane model, he found westward jets to undergo a series of mixed, baroclinic-barotropic instability, caused by the reversal of sign in the jet curvature $\partial^{2} u_{0} / \partial y^{2}$. Recall that $\beta \geqslant 0$. Hence, a barotropically unstable region, in which $\beta-\partial^{2} u_{0} / \partial y^{2}<0$, forms at the core of the westward jet (as is the case in run $\mathrm{W} 60 \mathrm{~N}$ ). The combined effects of vertical and horizontal shears reinforce each other to establish a mixed, baroclinicbarotropic unstable region. According to WKB analysis (e.g. Bender \& Orszag 1999), growing disturbances emanating from a westward jet are trapped (i.e. reflected) between two turning latitudes, initiating the sequence. Consistent with this, the meridional structure of the disturbance is able to remain close to the normal mode form. In contrast, disturbances emanating from the eastward jet are absorbed at or near the critical latitudes, resulting in a single cycle and meridional structure that changes with time.

Fig. 10b shows the equilibrated $\bar{u}$ and $\bar{\theta}$ at the end of the simulation. The original westward jet has been completely disrupted, giving way to a fairly barotropic eastward jet centred at $60^{\circ}$. Predominantly westward flow is now situated in the subtropics, at the upper levels of atmosphere. The reversal of the flow direction is consistent with E-P flux divergence shown in Fig. 11 (dotted line), which acts as a positive momentum source. Given that high-latitude 
9

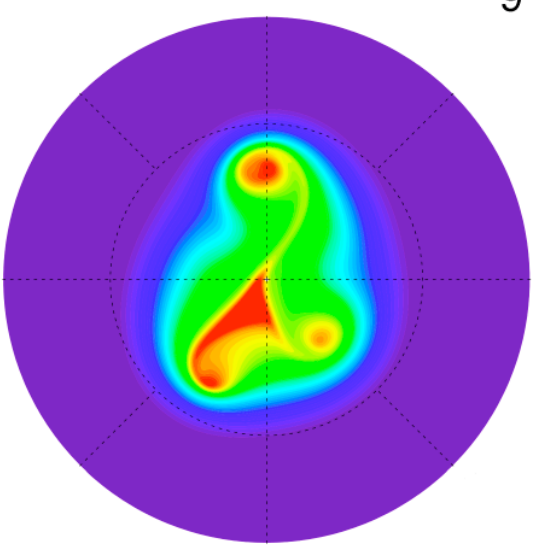

9

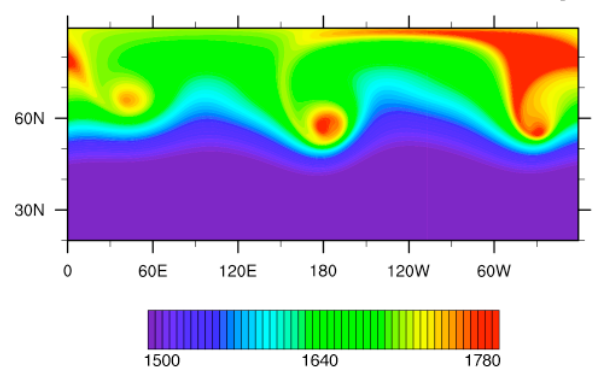

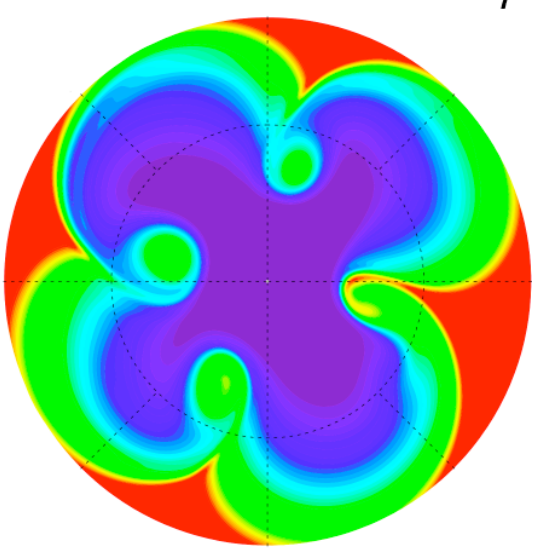

7

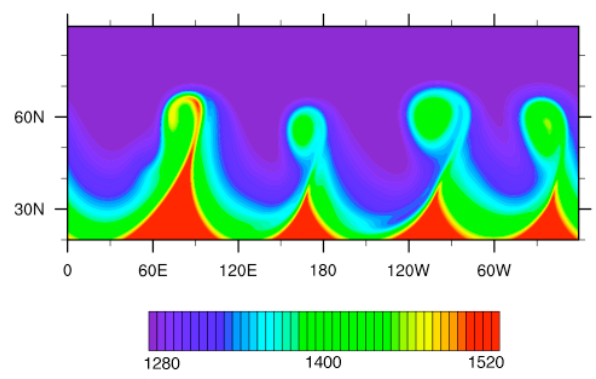

Figure 13. Temperature field $T$ for run $\mathrm{W} 60 \mathrm{~N}$ at $\tau=9$ (left) and $T$ for run E45N at $\tau=7$ (right). The fields are shown at 975 hPa pressure level. Top frame shows the field in polar stereographic view, centred on the north pole, and bottom frame shows the field in cylindrical-equidistant view, centred on the equator. In all frames area poleward of $\phi=20^{\circ}$ is shown. For run W60N the maximum and minimum values are $1500 \mathrm{~K}$ and $1780 \mathrm{~K}$, respectively. For run E45N the maximum and minimum values are $1280 \mathrm{~K}$ and $1520 \mathrm{~K}$, respectively. Contour interval is $6 \mathrm{~K}$ in both runs.

westward jets appear to be a fairly common feature in GCM simulations of hot giant extrasolar planets, the result here suggests external (e.g. stellar irradiation) or internal (e.g. wave) forcing may be required to maintain baroclinic westward jets. Note also from Figs. $10 \mathrm{~b}$ and 11, the negative zonal flow and E-P flux convergence, especially in the equatorial region. Significantly, such negative E-P flux divergences present a source of drag for equatorial jets. Finally, as in the 'paradigm case', the potential vorticity anomalies exceed $2 \Omega$ by over 12 per cent and by $\tau=35$ a cyclonic polar vortex forms that is warmer than its surroundings (not shown).

\subsection{Equatorial Jet}

The evolution of a broad, high-speed equatorial jet (run EEQ) is presented in this section. The initial flow and potential temperature is shown in Fig. 4c. Unlike the jets discussed in sections 3.3 and 3.4, the equatorial jet satisfies the Charney-Stern-Pedlosky instability criteria (iv) on its flanks (at $\sim 30^{\circ}$ on both northern and southern hemispheres), rather than at the core. The stability of this jet's core is consistent with linear theory of section 2 , which predicts no growth for a jet located equatorward of $28^{\circ}$.

Fig. 14 shows temperature $T$ and relative vorticity $\zeta$ fields at $\tau=26$. At this time the instability is well developed, with sharp fronts rolling up non-linearly into cyclones at $\phi \approx 35^{\circ}$, where the instability criteria is met. A mode with $\sim 7$ undulations can clearly be seen at this stage of the evolution. The number of undulations is significantly higher and the growth rate is significantly lower for EEQ than for simulations where the same jet is placed at $\phi=30^{\circ}$. Evidently, since the vertical shear of the equatorial jet at its flanks is significantly lower than at its core, a value smaller than the peak core value for the characteristic flow speed should be used in interpreting the results from the linear analysis. We have already seen that a weaker jet (shear) results in a smaller growth rate and wavelength of the most unstable mode at a given latitude (e.g. curves 'HD45L' and 'HD45' in Fig. 2). Hence, our non-linear calculations appear to be in qualitative agreement with linear theory. Despite the instability at the flanks, the core of the jet in run EEQ remain stable throughout the integration (up to $\tau=60$ ), in very good agreement with linear theory.

The EKE evolution for run EEQ is shown in Fig. 7 (solid line). The equatorial jet instability is shallow and confined to a pressure range between 1 to 0.7 bar, unlike the high-latitude jet instability; in those cases, the range of instability is much larger, extending up to 0.01 bar. Thus, only the lower pressure levels exhibit an increase in $E K E$ during the linear stage. For this reason, the EKE values have been multiplied by a factor of 50 in the figure: the globally averaged $E K E$ for run EEQ is much lower than for the 'paradigm case' or run W60N. Qualitatively, the non-linear evolution of run EEQ is much like that of run E45N, with waves tilting and breaking in same directions. However, potential vorticity anomaly only 


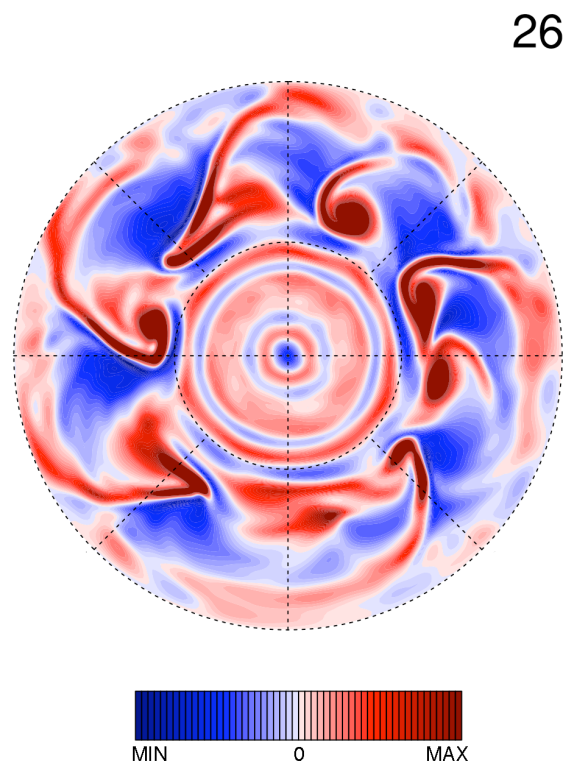

Figure 14. Temperature $T$ (left) and relative vorticity $\zeta$ (right) for run EEQ in polar stereographic view, centred on the north pole. The fields are shown at $975 \mathrm{hPa}$ pressure level for $\tau=26$. Maximum and minimum values for temperature are $1460 \mathrm{~K}$ and $1600 \mathrm{~K}$, respectively, with contour interval $3.5 \mathrm{~K}$. Values for $\zeta$ are in the range $\pm 1.5 \times 10^{-5} \mathrm{~s}^{-1}$, with contour interval $6 \times 10^{-7} \mathrm{~s}^{-1}$.

slightly exceeds the polar value and the cyclonic drift does not ensue. Therefore, a monolithic polar vortex does not form in this case.

Interestingly, the jet structure is only slightly altered by baroclinic instability from the basic state zonal flow. Mainly, the jet has become more barotropic at the flanks. This can be seen in Fig. 10c, which shows $\bar{u}$ and $\bar{\theta}$ at $\tau=60$. Relatively small values of E-P flux convergence equatorward of $30^{\circ}$ (see the solid line in Fig. 11) do not significantly contribute to the deceleration of the zonal mean zonal wind.

It is also worth noting that we have investigated stability properties of the westward equatorial jet and found it to be stable to baroclinic instability, in good agreement with Wang (1989). A westward jet placed at the equator would have to exceed sound speed, if the condition (8) of section 2 is to be fulfilled. However, we note that a broad, 'supersonic' westward jet does not appear to be unstable in full, non-linear GCM calculations (Thrastarson, private communication). But, the calculation is at $\mathrm{T} 21$ resolution (see next section). We have found eastward equatorial jets to be stable to baroclinic instability, if the width of the jet is $50^{\circ}$ and smaller.

\subsection{Numerical Convergence}

Baroclinic instability in numerical simulations of hot extrasolar planets is highly sensitive to numerical resolution (both horizontal and vertical) and to dissipation. High resolution is required to capture the instability accurately. In particular, for the jet profiles used, five or more layers is necessary to capture the instability, and good convergence is reached only with $\sim 10$ or more layers. In addition, high horizontal resolution ( $\gtrsim \mathrm{T} 85$ ) is necessary to ensure accurate representation of the eddy fluxes, as well as convergence. Separately, artificial viscosity must not be too high, as it results in artificially-enhanced stabilization of the baroclinic modes. We emphasise that resolution and dissipation requirements are dependent on the jet profile. Hence, the requirements should be carefully assessed for each profile employed. This 'problem-dependence' con- clusion has also been discussed by Thrastarson \& Cho (2011) for 'spin-up' experiments of hot giant planet circulations.

Before presenting the results, a brief discussion concerning our general approach to convergence testing is in order. In general, for numerical stability reasons, the usual practice is to use a larger dissipation coefficient value when performing a calculation with lower resolution - or, alternatively, a smaller coefficient value when performing the same calculation at higher resolution - so that the damping time is same at the truncation scale. This results in a different damping time for a given mode at different resolutions. However, here our aim is to demonstrate convergence of the numerical model. Hence, we employ the same value of the coefficient at all resolutions so that each mode, up to the truncation, experiences same dissipation rate in all the runs. The employed value is: $\nu=6 \times 10^{19} \mathrm{~m}^{4} \mathrm{~s}^{-1}$. Similar methodology has been implemented in e.g. Polvani et al. (2004) to test convergence in the Earth case. Later, we also demonstrate non-convergence when the damping time is chosen such that it is same at the truncation scale for all resolutions, as in the usual practice.

The requirement of adequate resolution is demonstrated in Fig. 15. The figure shows a set of four simulations with all parameters identical to run EEQ presented in section 3.5 - except the resolution. The resolutions are: T21 (a), T42 (b), T85 (c) and T170 (d). Note, panel (d) is run EEQ. All four runs use the same value of superviscosity coefficient, $\nu=6 \times 10^{19} \mathrm{~m}^{4} \mathrm{~s}^{-1}$, as already mentioned. Note also that the resolutions correspond, respectively, to $64 \times 32,128 \times 64,256 \times 128$ and $512 \times 256$ Gaussian grids in physical space. But, because of the exponential convergence property of the spectral method, they are equivalent in accuracy to finite difference grids $\mathcal{O}(10)$ times finer in resolution (e.g. Thrastarson \& Cho 2011). Polar stereographic projections of the relative vorticity field $\zeta$ at $\tau=22$ are shown, when the instability is in the early exponentially-growing stage (see Fig. 7).

Visual inspection of the fields readily reveals that the T21 (a) and T42 (b) runs do not converge to the T170 (d) run. The T85 (c) 
a)

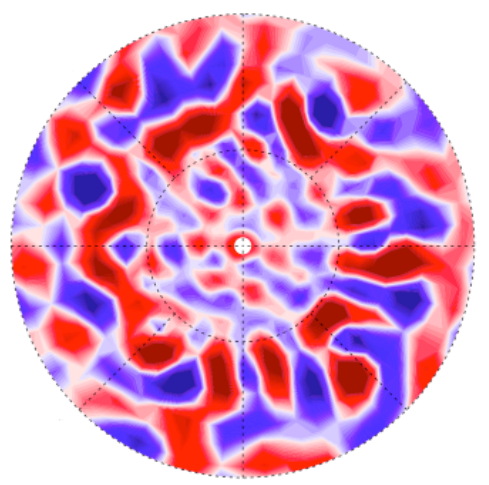

c)

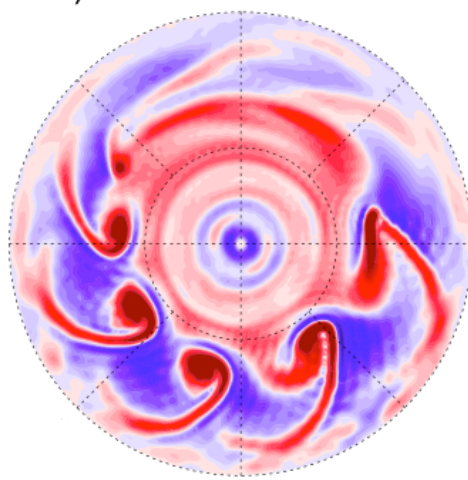

T85 b)

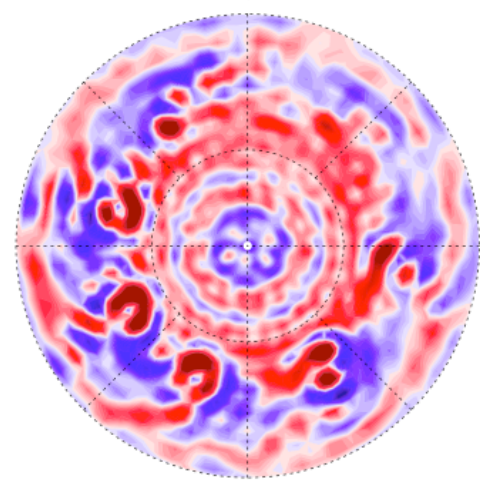

d)

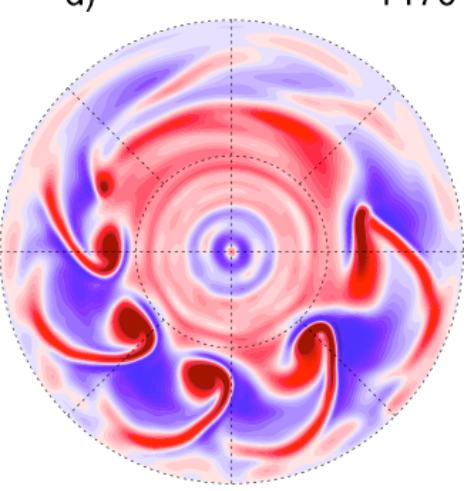

T170

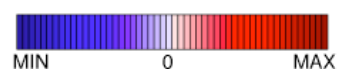

Figure 15. Polar stereographic view of the relative vorticity field $\zeta$, centred on the north pole, for four runs with all parameters identical - except the horizontal resolution. The common parameters are as in run EEQ. The fields at $\tau=22$ are shown. The number at upper right in each panel indicates the resolution. Contour levels are the same to those in Fig. 14.
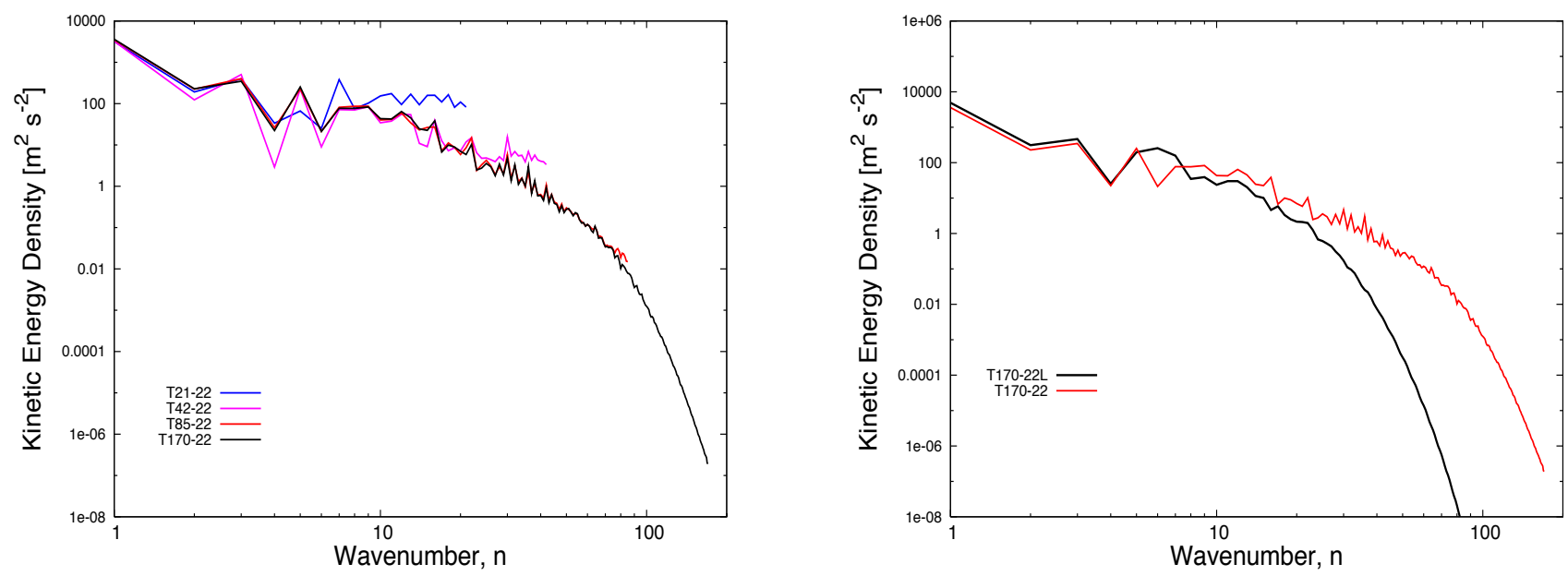

Figure 16. Kinetic energy density $\left[\mathrm{m}^{2} \mathrm{~s}^{-2}\right.$ (per wave number)] as a function of (total) wavenumber $n$. Spectra for the fields from the four runs shown in Fig. 15 (left). The different lines refer to different horizontal resolutions, as indicated in the legend. The viscosity coefficient is same $\left(\nu=6 \times 10^{19} \mathrm{~m}^{4} \mathrm{~s}^{-1}\right)$ in all four runs. Spectra for run EEQ at T170 resolution with different viscosity coefficients (right): $\nu=6 \times 10^{19} \mathrm{~m}^{4} \mathrm{~s}^{-1}$ (red line) and $\nu=10^{21} \mathrm{~m}^{4} \mathrm{~s}^{-1}$ (black line), respectively. 
a)

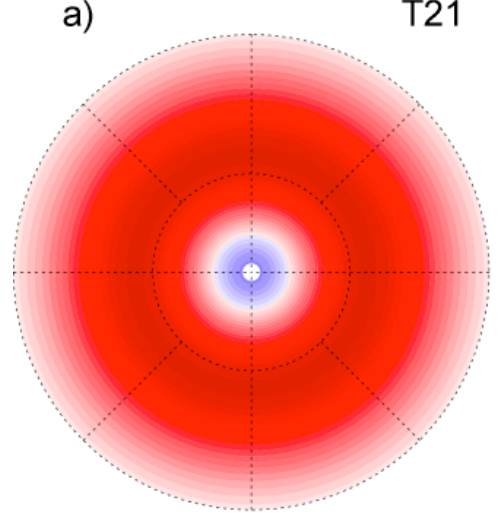

c)

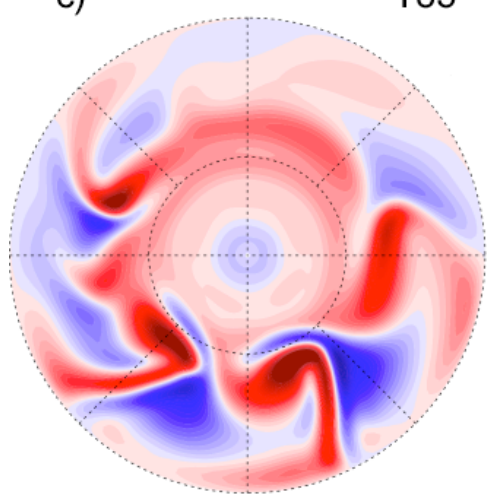

b)

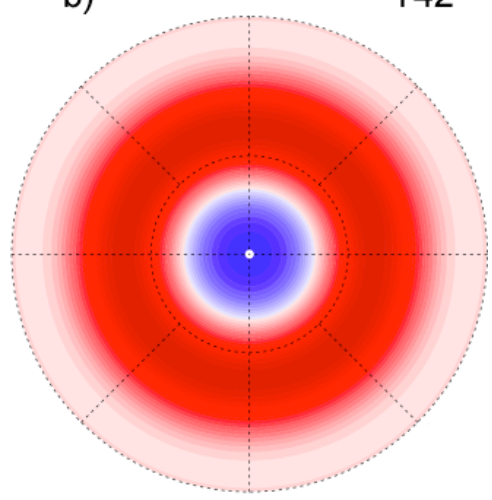

d)

$\mathrm{T} 42$
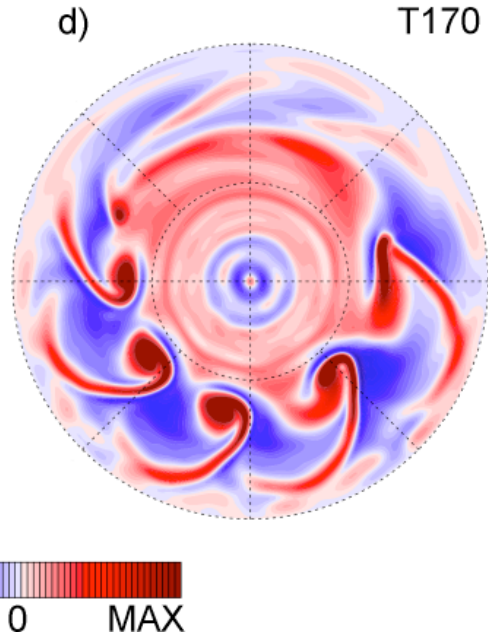

Figure 17. Same as Fig. 15 but with $\tau_{\mathrm{d}}^{-1}=5.07 \times 10^{-4} \mathrm{~s}^{-1}$ in all runs. Maximum and minimum values for frames a) and b) are $\pm 3 \times 10^{-6} \mathrm{~s}^{-1}$ and for frames c) and d) $\pm 1.5 \times 10^{-5} \mathrm{~s}^{-1}$; the contour intervals are, respectively, $1.4 \times 10^{-7} \mathrm{~s}^{-1}$ and $6 \times 10^{-7} \mathrm{~s}^{-1}$.

run is marginally converged, though this may change after a long time (e.g. many hundreds of planetary rotations). In the figure, frames (a) and (b) are qualitatively different than frames (c) and (d), which clearly show mode- 6 instability. The T85 run in frame (c) captures the basic structure present in the T170 run in frame (d). However, spurious small-scale oscillations are also clearly visible in frame (c); these are not present in frame (d). The small-scale oscillations contaminate the calculation - causing the calculation to blow up, depending on the numerical parameters used; see e.g. discussion in Thrastarson \& Cho (2011).

The above behavior can be quantified by computing the corresponding kinetic energy spectra for each run. The spectra for the fields shown in Fig. 15 are presented in the left panel of Fig. 16. Inspection of the T85 and T170 spectra (red and black lines, respectively) confirms the convergence of the simulations. Note the presence of a clear dissipation range in the T170 run. In contrast, the appearance of nearly grid-scale waves in physical space for $\mathrm{T} 21$ and $\mathrm{T} 42$ resolution runs corroborates the tendency of the spectrum (blue and pink lines for T21 and T42, respectively) in these runs to peel off and curl up considerably left of the aliasing limit $(\sim 21$ for $\mathrm{T} 21$ and $\sim 42$ for T42). This is caused by discretization errors that are not adequately controlled by the applied explicit viscosity.

We have also performed an analogous series of runs in which a much larger dissipation coefficient value, $\nu=10^{21} \mathrm{~m}^{4} \mathrm{~s}^{-1}$ has been used. This mimics 'properly' dissipated runs at T21 and T42 resolutions (i.e. runs with well represented dissipation range in spectral space). However, in this series the high resolution calculations are significantly over-dissipated and the physical space picture is characterised by a severe reduction in eddy kinetic energy at all times. This is supported by the spectra for two T170 resolution simulations with the two coefficients (right frame of Fig. 16). The spectrum for a run using $\nu=10^{21} \mathrm{~m}^{4} \mathrm{~s}^{-1}$ (black line) is shown together with the spectrum of the previously presented $\mathrm{T} 170$ run with $\nu=6 \times 10^{19} \mathrm{~m}^{4} \mathrm{~s}^{-1}$ (red line). With the larger $\nu$, the spectrum is severely over-dissipated with only $\sim 20$ modes being resolved; the rest of the modes clearly lie in the dissipation range. In contrast, at least $\sim 80$ modes are well-resolved with the smaller $\nu$ value.

It is important to understand that wavenumbers short-ward of the fiducial 'dissipation range' (i.e. less than 20 and 80, respectively, in the runs discussed above) are still affected by a small amount of dissipation in practice: that is, dissipation affects the entire spectrum of wavenumbers continuously, rather than just the wavenumbers in the dissipation range. The amount, while small, can nevertheless be dynamically significant, as it can change the quantitative character of the instability, even suppress the instability altogether. Indeed, if the value of $\nu$ is increased further, to $10^{22} \mathrm{~m}^{4} \mathrm{~s}^{-1}$, the baroclinic waves completely disappear.

As discussed above, a common practice in numerical studies which vary the resolution is to adjust the dissipation coefficient $\nu$ so that the damping time $\tau_{\mathrm{d}}$ is same at the truncation scale $n_{t}$ :

$$
\tau_{\mathrm{d}}=\frac{1}{\nu}\left[\frac{R_{p}^{2}}{n_{t}\left(n_{t}+1\right)}\right]^{2} .
$$


While the physical basis of this procedure is arguable, we demonstrate here that the practice still does not lead to convergence at low resolution. Consider the value, $\nu=6 \times 10^{19} \mathrm{~m}^{4} \mathrm{~s}^{-1}$, used in the high resolution simulation discussed above. The dissipation rate at the truncation scale for HD209458b corresponding to this $\nu$ value is $\tau_{\mathrm{d}}^{-1}=5.07 \times 10^{-4} \mathrm{~s}^{-1}$. Note that this damping rate is comparable to the rates used in current flow modeling studies of hot gaseous extrasolar planet atmospheres at resolutions lower than T170 (e.g. Rauscher \& Menou 2010; Thrastarson \& Cho 2010, 2011; Heng, Menou \& Phillips 2011). Its significance can be seen in Fig. 17.

Fig. 17 shows the effects of adjusting $\nu$ so that the damping rate is constant at the truncation scale. The rate used is the one just discussed above: $\tau_{\mathrm{d}}^{-1}=5.07 \times 10^{-4} \mathrm{~s}^{-1}$. Relative vorticity field $\zeta$ at four resolutions for run EEQ at $\tau=22$ is shown. Two points are clear from the figure. First, the simulations in this series are not converged. The $\mathrm{T} 21$ and $\mathrm{T} 42$ resolution runs are completely over-dissipated and the momentum and heat transports are absent throughout the duration of the runs, up to $\tau=60$. Second, the $\nu$ values used in current GCM modelling studies of hot extrasolar giant planets do not permit the instability. Note that, if the dissipation rate is chosen instead to be the one that 'adequately' permits the instability in the low resolution run, the high resolution runs are severely under-dissipated and inundated with noise (not shown). Either way, convergence is not achieved by fixing the dissipation rate at the truncation scale.

Arguably, the two points above may not be significant for atmospheres characterized by a very short diabatic relaxation time. For then the thermal damping would dominate and naturally shortcircuit the above issues pertaining to the artificial viscosity and resolution. However, in some GCM simulations, the dynamicallyrelevant, intrinsic thermal relaxation time is not always short after quasi-equilibration is reached, even above the $\sim 1$ bar level $^{4}$ (Thrastarson (private communication)). Moreover, the instabilities at higher latitudes possess short growth times and are much less affected by short relaxation times. Additionally, there is the issue of transient growth, which we have not discussed in this work. The non-normal modes, associated with such growth, may operate on a much shorter time scale than the growths described in this work.

We stress here that the high resolution runs described do not merely contain more fine-scale structures that presumably do not significantly affect the evolution. On the contrary, we have found that high resolution fundamentally affects the evolution. For example, bulk eddy heat- and momentum-fluxes differ significantly (up to an order of magnitude) in high- and low-resolution simulations. The reason for this is that vorticity anomalies (eddies) are much stronger, in addition to the filaments, in the high-resolution simulations; and, EKE growth is exponential in the stage of the evolution when these structures have emerged (Fig. 7). Hence, heat and momentum are redistributed much more effectively.

\section{SUMMARY AND DISCUSSION}

Baroclinic instability on extrasolar planets has not been studied thus far. In this work we have used an advanced pseudospectral GCM to perform an extensive study of the stability and non-linear evolution of balanced jets on hot extrasolar planets. Our non-linear baroclinic instability calculations have been fully validated against previous similar calculations for the Earth (e.g. Polvani et al. 2004;

${ }^{4}$ It is, in general, sufficiently long below this level.
Jablonowski \& Williamson 2006). For concreteness, we have presented here results for a model planet with physical parameters corresponding to the close-in giant planet HD209458b and focused on the stability of high-speed (typically $1000 \mathrm{~m} \mathrm{~s}^{-1}$ ) eastward jets at the equator and westward jets at high latitudes. Broad jets of such magnitude are a common feature in current GCM simulations of tidally-synchronized giant planet atmospheres.

We have derived linear growth rate and phase speed spectra, via standard normal mode analysis, and compared the results with full non-linear numerical simulations. According to our linear analysis of the two-layer primitive equations model on the $\beta$-plane, the growth rate of the instability is reduced for a jet located at low latitudes, compared with a jet located at high latitudes. Near the equator, where the deformation length scale $L_{\mathrm{D}}$ becomes too large to accommodate baroclinic waves, the linear theory predicts stability. In general, linear analysis agrees reasonably well with the full nonlinear calculations at the early stage of the unstable evolution, during the transient phase. After a long time, in simulations with high values of initial potential vorticity anomaly (i.e. $\left|q^{\prime} / 2 \Omega\right| \gtrsim 1.2$, where $q^{\prime}$ is the anomaly), cyclones merge to form robust monolithic vortices at the poles. This is not captured by linear analysis.

As expected, full non-linear calculations show richer behavior than that obtained through linear analysis. Non-linear simulations show that baroclinic instability occurs for all eastward jet profiles used in this study. In particular, broad equatorial eastward jets are unstable (on a time scale of $\sim 20$ planetary rotations), despite stability suggested by the linear analysis. The instability takes place at the jet flanks, where there is still a significant vertical shear to satisfy the necessary condition for instability. The jet core is stable, unlike in the jets situated at higher latitudes; this is in accordance with linear theory. Westward jets near the equator, however, remain stable, both at the core and the flanks. To the best of our knowledge this is the first time non-linear baroclinic instability has been studied for a broad equatorial jet in the atmosphere. In general, we have found westward jets to be more stable compared to their eastward counterparts (e.g. at midlatitude, instability timescale of $\sim 6$ planetary rotations for westward jets vs. $\sim 3$ planetary rotations for eastward jets), and to require much stronger vertical shear for instability in the full primitive equations system. Additionally, we have demonstrated in this work that baroclinic instability does not require a solid boundary on planets, as long as there is a change of sign in the meridional potential vorticity gradient $\partial q_{0} / \partial \phi$ in the domain's interior.

By performing the simulations described above with a wide range of horizontal resolution (from T21 to T170), we have found that the calculations do not converge for resolutions below T85. This is a somewhat stronger requirement than for Earth simulations and is primarily due to the much stronger jet amplitude on hot extrasolar planets $\left(\sim 1000 \mathrm{~m} \mathrm{~s}^{-1}\right.$, compared to $\sim 50 \mathrm{~m} \mathrm{~s}^{-1}$ for the Earth). Furthermore, we have found that baroclinic instability does not occur at all if the artificial viscosity coefficient used in the calculation is too high. A high artificial viscosity is often used to stabilize numerical simulations against strong forcing in current studies of extrasolar planet atmospheres. Given this, baroclinic instability is unlikely to be represented in current simulations - even when necessary conditions for instability are satisfied. This may pose a serious issue in flow modelling studies of extrasolar planet atmospheres in which the natural diabatic relaxation time is not too short (i.e. greater than a few planetary rotations).

The results presented in this paper show that baroclinic instability is significant for understanding characteristics of hot extrasolar planets which possess fast jet streams. This instability is likely 
to play a role in weather, general circulation and large scale variability of a few to few tens of planetary rotation periods on these planets (Cho 2008). For example, the process could generate large long-lived storms that could be observed remotely. Sharp fronts produced in baroclinic instability life-cycles can also act as a gravity wave source (e.g. O'Sullivan \& Dunkerton 1995; Plougonven \& Snyder 2007). Gravity waves are expected to play an important role in stably stratified atmospheres of hot extrasolar planets: they can modify the circulation through exerting accelerations (positive and negative) on the mean flow, as well as transporting heat vertically from deep regions to sensible regions and laterally from day side to night side (Watkins \& Cho 2010).

In this work, we only discuss adiabatic calculations. Adiabatic calculations are important to cleanly delineate many subtle effects in rotating-stratified fluid that could obscure baroclinic instability. They are also important as a foundation for the instability under forced conditions, which require careful study. The complex effects of forcing on the background flow itself remains to be elucidated. We have also focused mainly on the instability and subsequent evolution of jets in isolation and only lightly touched on the effect of concomitant eddies on the background flow. In summary, the full effect of baroclinic instability on the mean flow on hot extrasolar planets remains to be carefully studied. Some of the issues identified here will be addressed in future work.

\section{ACKNOWLEDGMENTS}

The authors thank Heidar Thrastarson and Chris Watkins for useful discussions and the reviewer for helpful comments. This work is supported by a research studentship from the Westfield Trust to I.P. and the Science and Technology Facilities Council grant PP/E001858/1 and the Westfield Small Grant to J.Y-K.C.

\section{APPENDIX A: NON-DIMENSIONAL STABILITY ANALYSIS}

Equations set (6) is made non-dimensional by introducing the 'discretized deformation length scale', horizontal length scale, timescale, and height scale:

$$
\begin{aligned}
\hat{L}_{\mathrm{D}} & =\frac{1}{f_{0}} \sqrt{h_{2} \triangle p \sigma_{0}}, \\
L & =\frac{1}{\sqrt{2}} \hat{L}_{\mathrm{D}}, \\
T & =\frac{1}{\sqrt{2}}\left(\hat{L}_{\mathrm{D}} / U_{0}\right), \\
H & =\frac{1}{\sqrt{2}}\left(U_{0} f_{0} \hat{L}_{\mathrm{D}}\right),
\end{aligned}
$$

respectively. Hence, the set of equations becomes characterised only by the Rossby number,

$$
\hat{R} o=\frac{U_{0} \sqrt{2}}{f_{0} \hat{L}_{\mathrm{D}}},
$$

and the Charney-Green number,

$$
\hat{\gamma}=\frac{\beta \hat{L}_{\mathrm{D}}^{2}}{2 U_{0}} .
$$

The Charney-Green number measures the relative importance of the planetary vorticity gradient to the relative vorticity gradient. Using these non-dimensionalised scales and parameters, the equations now read:

$$
\begin{aligned}
\frac{\partial}{\partial \tilde{t}}\left(\frac{\partial^{2} \tilde{\Psi}}{\partial \tilde{x}^{2}}\right) & =-\frac{\partial}{\partial \tilde{x}}\left(\frac{\partial^{2} \tilde{\Theta}}{\partial \tilde{x}^{2}}\right)-\hat{\gamma} \frac{\partial \tilde{\Psi}}{\partial \tilde{x}} \\
\frac{\partial}{\partial \tilde{t}}\left(\frac{\partial^{2} \tilde{\Theta}}{\partial \tilde{x}^{2}}\right) & =-\frac{\partial}{\partial \tilde{x}}\left(\frac{\partial^{2} \tilde{\Psi}}{\partial \tilde{x}^{2}}\right)-\frac{1}{\hat{R}_{o}} \frac{\partial^{2} \tilde{\chi}}{\partial \tilde{x}^{2}}-\hat{\gamma} \frac{\partial \tilde{\Theta}}{\partial \tilde{x}} \\
\frac{\partial}{\partial \tilde{t}}\left(\frac{\partial^{2} \tilde{\chi}}{\partial \tilde{x}^{2}}\right) & =\frac{1}{\hat{R} o} \frac{\partial^{2} \tilde{\Theta}}{\partial \tilde{x}^{2}}-\frac{1}{\hat{R} o} \frac{\partial^{2} \tilde{\Phi}}{\partial \tilde{x}^{2}}-\hat{\gamma} \frac{\partial \tilde{\chi}}{\partial \tilde{x}} \\
\frac{\partial \tilde{\Phi}}{\partial \tilde{t}} & =\frac{\partial \tilde{\Psi}}{\partial \tilde{x}}-\frac{1}{\hat{R o}} \frac{\partial^{2} \tilde{\chi}}{\partial \tilde{x}^{2}},
\end{aligned}
$$

where $(\tilde{\sim})$ denotes non-dimensional variables. The variables, $(\tilde{\Psi}, \tilde{\Theta}, \tilde{\chi}, \tilde{\Phi})$, are the non-dimensional counterparts of $\left(\psi_{+}^{\prime}, \psi_{-}^{\prime}, \chi_{-}^{\prime}, \Phi_{-}^{\prime}\right)$ in section 2.3 .

Denoting disturbances by

$$
\tilde{\mathbf{\Psi}}=\hat{\tilde{\mathbf{\Psi}}} \exp \{i \tilde{k}(\tilde{x}-\tilde{c} \tilde{t})\}
$$

where $\tilde{\Psi}=(\tilde{\Psi}, \tilde{\Theta}, \tilde{\chi}, \tilde{\Phi})^{\mathrm{T}}, \hat{\tilde{\Psi}}=(\hat{\tilde{\Psi}}, \hat{\tilde{\Theta}}, \hat{\tilde{\chi}}, \hat{\tilde{\Phi}})^{\mathrm{T}}$, and $\tilde{c} \in \mathbb{C}$, equations (A1) reduce to

$$
\tilde{\mathbf{M}} \hat{\tilde{\Psi}}=0
$$

where

$$
\tilde{\mathbf{M}}=\left[\begin{array}{cccc}
-\tilde{c}-\hat{\gamma} / \tilde{k}^{2} & 1 & 0 & 0 \\
1 & -\tilde{c}-\hat{\gamma} / \tilde{k}^{2} & -i /(\tilde{k} \hat{R} o) & 0 \\
0 & i /(\tilde{k} \hat{R} o) & -\tilde{c}-\hat{\gamma} / \tilde{k}^{2} & -i /(\tilde{k} \hat{R} o) \\
1 & 0 & -i \tilde{k} / \hat{R} o & \tilde{c}
\end{array}\right]
$$

This leads to a normal mode solution fulfilling the fourth order characteristic equation for $\tilde{c}$ :

$$
\begin{aligned}
& \tilde{c}^{4}+\tilde{c}^{3}\left(\frac{3 \hat{\gamma}}{\tilde{k}^{2}}\right)+\tilde{c}^{2}\left(\frac{3 \hat{\gamma}^{2}}{\tilde{k}^{4}}-\frac{1}{\hat{R o}^{2}}-\frac{1}{\tilde{k}^{2} \hat{R o}}-1\right)+ \\
& \tilde{c}\left(\frac{\hat{\gamma}^{3}}{\tilde{k}^{6}}-\frac{2 \hat{\gamma}}{\tilde{k}^{2} \hat{R o}^{2}}-\frac{\hat{\gamma}}{\tilde{k}^{4} \hat{R o}^{2}}-\frac{\hat{\gamma}}{\tilde{k}^{2}}\right)+ \\
& \frac{1}{\hat{R o}^{2}}\left(1-\frac{1}{\tilde{k}^{2}}-\frac{\hat{\gamma}^{2}}{\tilde{k}^{4}}\right)=0 .
\end{aligned}
$$

Equation (A2) is solved numerically for varying values of $\hat{R} o$ and $\hat{\gamma}$. Fig. A1 shows the results for HD209458b (cf. Fig. 2).

Non-dimensional analysis is useful because it explicitly gives the dependence of stability properties on dynamically-significant non-dimensional numbers, such as $\hat{R} o$ and $\hat{\gamma}$. Extensive exploration of the solutions for a continuum of $\hat{R} o$ and $\hat{\gamma}$ values reveals that, as $\hat{R} o$ and/or $\hat{\gamma}$ increase, the low wavenumber cutoff for instability increases while the high wavenumber cutoff for instability and growth rate decrease slightly (Fig. A1). Fig. A2 illustrates how the growth rates depend on $\hat{R} o$ when $\hat{\gamma}$ is held fixed at a typical midlatitude value for HD209458b. As $\hat{R} o$ increases from $\hat{R} o<<1$ to $\hat{R} o \sim 1$, the growth rate decreases linearly. However, the reduction in growth rate is exponential as the $\hat{R} o \sim 1$ threshold is crossed and the two-layer linear analysis predicts stability for flows with $\hat{R o}>3.3$.

To obtain the dimensional values of the growth rate from Fig. A1 and Fig. A2, multiply the growth rate $(\tilde{k} \cdot \Im \mathfrak{m}\{\tilde{c}\})$, for example, by $U_{0} \sqrt{2} / \hat{L}_{\mathrm{D}}$ and the wavenumber $(\tilde{k})$ by $\sqrt{2} / \hat{L}_{\mathrm{D}}$. The result of Fig. A1 is growth rates identical to those presented in Fig. 2. The dimensional phase speed is obtained similarly. 


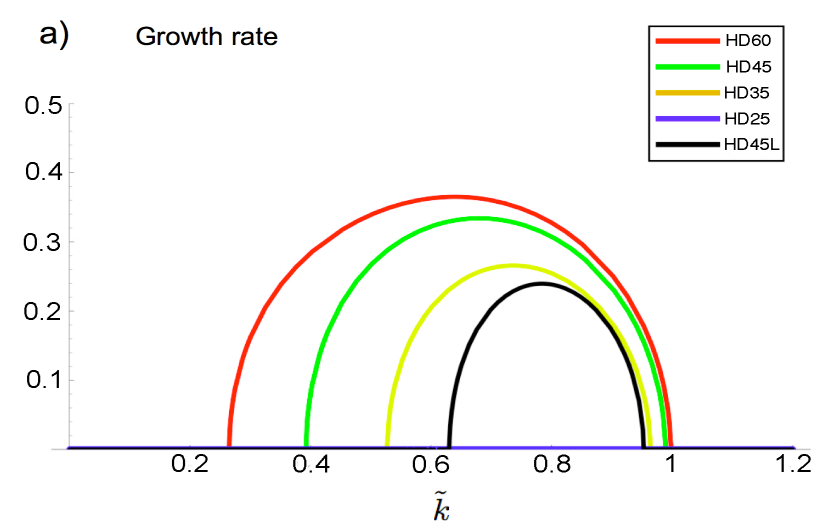

b) Phase Speed

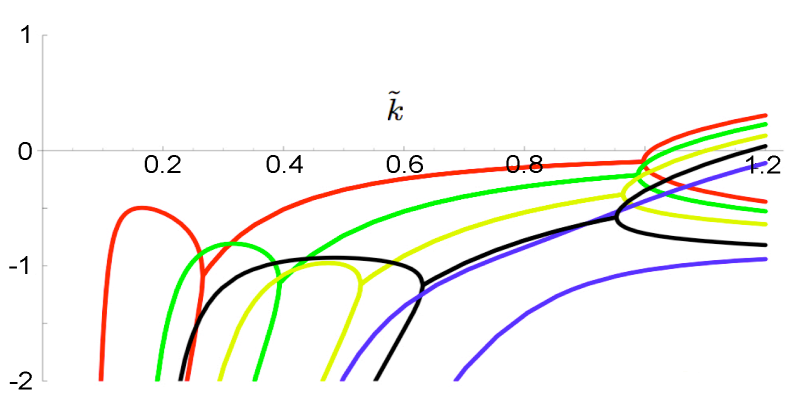

Figure A1. Non-dimensional growth rate $[\tilde{k} \cdot \Im \mathfrak{m}\{\tilde{c}\}]$ (left) and phase speed [ $\Re \mathfrak{e}\{\tilde{c}\}]$ (right) for HD209458b, as a function of non-dimensional wavenumber $\tilde{k}$. Curves 'HD60', 'HD45', 'HD35' and 'HD25' represent growth rates and phase speeds at $\phi=\left(60^{\circ}, 45^{\circ}, 35^{\circ}, 25^{\circ}\right)$ computed with $(\hat{R} o=0.76, \hat{\gamma}=0.14)$, $(\hat{R o}=0.76, \hat{\gamma}=0.3),(\hat{R} o=0.76, \hat{\gamma}=0.51),(\hat{R} o=0.76, \hat{\gamma}=1.04)$. Curve 'HD45L' has been computed for HD209458b parameters at $\phi=45^{\circ}$, but with $U_{0}=200 \mathrm{~m} \mathrm{~s}^{-1}$ corresponding to $(\hat{R} o=0.3, \hat{\gamma}=0.72)$. To obtain dimensional values, multiply the growth rate by $U_{0} \sqrt{2} / \hat{L}_{\mathrm{D}}$ and wavenumber by $\sqrt{2} / \hat{L}_{\mathrm{D}}$.

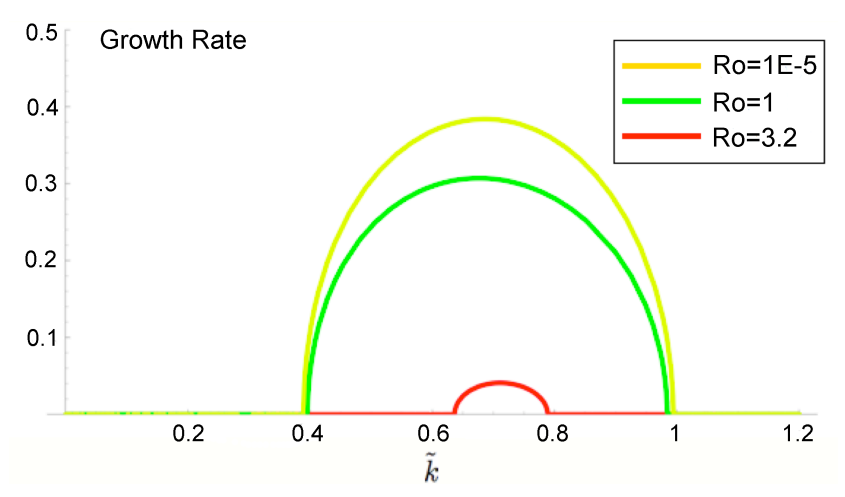

Figure A2. Non-dimensional growth rate $[\tilde{k} \cdot \Im \mathfrak{m}\{\tilde{c}\}]$ as a function of nondimensional wavenumber $\tilde{k}$ for different values of $\hat{R}$. Yellow, green and red curves have been calculated with $\hat{R} o=10^{-5}, \hat{R} o=1$ and $\hat{R} o=3.2$, respectively. The value of $\hat{\gamma}$ is held constant at $\hat{\gamma}=0.3$. To obtain dimensional values, multiply the growth rate by $U_{0} \sqrt{2} / \hat{L}_{\mathrm{D}}$ and wavenumber by $\sqrt{2} / \hat{L}_{\mathrm{D}}$.

\section{REFERENCES}

Andrews D. G., McIntyre M. E., 1978, J. Atmos. Sci., 35, 175 Asselin R., 1972, Mon. Wea. Rev., 100, 487

Bender C. M. \& Orszag S. A., 1999, 'Advanced Mathematical Methods for Scientists and Engineers' (Springer-Verlag, New York)

Canuto C., Hussaini M. Y., Qarteroni A., Zang T. A., 1988, Spectral Methods in Fluid Dynamics (New York, NY: Springer)

Charney J. G., 1947, J. Meteor., 4, 135

Charney J. G., Stern M. E., 1962, J. Atmos. Sci., 19: 159

Cho J. Y-K., 2008, Phil. Trans. Roy. Soc. A, 366, 4477

Cho J. Y-K., Polvani L., 1996, Phys. Fluids, 8, 6
Cho J. Y-K., Menou K., Hansen B. M. S., Seager S., 2003, ApJ, 587, L117

Cho J. Y-K., Menou K., Hansen B. M. S., Seager S., 2008, ApJ 675,817

Eady E. T., 1949, Tellus, 1, 33

Eliasen E., Mechenhauer B., Rasmussen E., 1970, Copenhagen Univ., Inst. Teoretisk Meteorologi, Tech. Rep. 2

Feldstein S. B., 1991, J. Atmos. Sci. , 48, 1701

Fraedrich K., Frisius T., 2001, Q. J. Roy. Met. Soc., 127, 2053

Gall R., 1976, J. Atmos. Sci., 33, 349

Green J. S. A., 1960, Q. J. Roy. Met. Soc., 86, 237

Guillot T., Showman A. P., 2002, A\&A, 385, 156

Heng K., Menou K., Phillips P. J., 2011, MNRAS, 413, 2380

Holton J. R., 1992, 'Introduction to Dynamic Meteorology' (Academic Press, San Diego)

Hoskins B. J., McIntyre M. E., Robertson A. W., 1985, Q. J. Roy. Met. Soc., 111, 877

Jablonowski C., Williamson D. L., 2006, Q. J. Roy. Met. Soc., 132, 2943

James I. N., 1987, J. Atmos. Sci., 44, 3710

Kasahara A., 1974, Mon. Wea. Rev., 102, 509

Kuo H. L., 1979, J. Atmos. Sci., 36, 2360

Menou K., Rauscher E., 2009, ApJ, 700, 887

Nakamura N., 1993, J. Atmos. Sci., 50, 357

Nakamura N., 1993, J. Atmos. Sci., 50, 2159

Orszag A., 1970, J. Atmos. Sci., 27, 890

O’Sullivan D., Dunkerton T. J., 1995, J. Atmos. Sci., 52, 3695

Pedlosky J., 1964, J. Atmos. Sci., 21 , 342

Pedlosky J., 1987, 'Geophysical Fluid Dynamics' (2nd ed., Springer-Verlag, New york)

Phillips N. A., 1951, J. Meteor., 8, 381

Plougonven R., Snyder C., 2007, J. Atmos. Sci., 64, 2502

Polvani L. M., Scott R. K., Thomas S. J., 2004, Mon. Wea. Rev., 132, 2539

Rauscher E., Menou K., 2010, ApJ, 714, 1334

Robert A., 1966, J. Met. Soc. Japan, 44, 237

Scott R. K., 2011, Geophys. Astrophys. Fluid Dyn., 105, 409 
Scott R. K., Rivier L., Loft R., Polvani L. M, 2003, NCAR Technical Note

Sharp D. H., 1984, Physica D, 12, 3

Showman A. P., Cooper C. S., Fortney J. J., Marley M. S., 2008, ApJ, 682, 559

Showman A. P., Cho J. Y-K., Menou K., 2010, in EXOPLANETS, ed., S. Seager, Tuscon: University of Arizona Press

Simmons A. J., Hoskins B. J, 1979, J. Atmos. Sci., 36, 1239

Simons T. J., 1972, J. Atmos. Sci., 8, 392

Thorncroft C. D., Hoskins B. J., McIntyre M. E., 1993, Q. J. Roy. Met. Soc., 119, 17

Thrastarson H. Th., Cho J. Y-K., 2010, ApJ, 716, 144

Thrastarson H. Th., Cho J. Y-K., 2011, ApJ, 729, 117

Vallis G. K., 2006, 'Atmospheric and Oceanic Fluid Dynamics' (Cambridge University Press, Cambridge)

Wiin-Nielsen A., 1963, Tellus, 15, 1, 1

Watkins C., Cho J. Y-K., 2010, ApJ, 714, 904

Wang B., 1989, Tellus, 42A, 463 